NASATM- $-.77-207568$

\title{
REDUCTION OF HELICOPTER BLADE-VORTEX INTERACTION NOISE BY ACTIVE ROTOR CONTROL TECHNOLOGY
}

\author{
Yung H. Yu, ${ }^{*}$ Bernd Gmelin, ${ }^{\dagger}$ Wolf Splettstoesser, ${ }^{\dagger}$ Jean J. Philippe, ${ }^{\ddagger}$ Jean Prieur ${ }^{\ddagger}$ \\ and Thomas F. Brooks \\ * Aeroflightdynamics Directorate, AVRDEC, Ames Research Center, Moffett Field, CA 94035-1000. U.S.A.; \\ ${ }^{+}$Deutsche Forschungsanstalt fir Luft- und Raumfahrt (DLR). Braunschweig. Germany; \\ \$ Office National D'Etudes et de Recherches Aerospatiales (ONERA). Chatillon, France: \\ "National Aeronautics and Space Administration (NASA), Langley Research Center, Hampton. VA. U.S.A.
}

(Received for publication 30 May 1997)

\begin{abstract}
Helicopter blade-vortex interaction noise is one of the most severe noise sources and is very important both in community annoyance and military detection. Research over the decades has substantially improved basic physical understanding of the mechanisms generating rotor blade-vortex interaction noise and also of controlling techniques, particularly using active rotor control technology. This paper reviews active rotor control techniques currently available for rotor blade-vortex interaction noise reduction. including higher harmonic pitch control, individual blade control, and on-blade control technologies. Basic physical mechanisms of each active control technique are reviewed in terms of noise reduction mechanism and controlling aerodynamic or structural parameters of a blade. Active rotor control techniques using smart structures/materials are discussed, including distributed smart actuators to induce local torsional or flapping deformations. Published by Elsevier Science Lid
\end{abstract}

\section{CONTENTS}

1. INTRODUCTION

2. BVI NOISE CHARACTERISTICS

3. ACTIVE ROTOR CONTROL CONCEPTS

3.1. Blade root control concepts: $\mathrm{HHC}$ and $1 \mathrm{BC}$

3.2. Controller design

4. HHC AND IBC FOR BVI NOISE REDUCTION

4.1. HHC investigations $\quad 665$

4.2. IBC investigations $\quad 676$

5. ANALYSIS OF BVI PHENOMENA AND NOISE WITH HHC 678

6. SMART ACTIVE ROTOR CONTROL CONCEPTS FOR BVI NOISE REDUCTION 682

7. CONCLUSION

REFERENCES

\section{NOTATION}

ARES --aeroelastic rotor experimental system

BL---baseline case (no HHC)

$C_{\mathrm{T}}$ - rotor thrust coefficient

$C_{\mathrm{p}}-$ pressure coefficient

$\mathrm{dBA}-\mathrm{A}$-weighted noise level

EPNdB--effective perceived noise level

LLS - laser-light sheet

$\mathrm{MN}$--minimum noise $\mathrm{HHC}$ case

$\mathrm{MV}$-minimum vibration $\mathrm{HHC}$ case

$M_{11}$ hover tip Mach number

\author{
TDT--transonic dynamics tunnel \\ TPNdB - tone perceived noise level \\ $\alpha$-rotor tip path plane angle referenced to tunnel \\ streamwise axis, positive for backward tilt (deg.) \\ $x_{S}-$ rotor shaft angle (deg.) \\ $x_{\text {TPP }}$ tip-path-plane angle \\ $\theta_{c}-\cdots$ amplitude of higher harmonic pitch (deg.) \\ $\mu-$ advanced ratio \\ $\psi$-blade azimuthal angle (deg.) \\ $\psi_{\mathrm{c}}-$-blade azimuthal angle selected for $\theta_{\mathrm{c}}$ (deg.)
}

\section{INTRODUCTION}

Helicopter impulsive noise is known to originate from two distinct aerodynamic events: the compressible flow field due to high tip Mach number on the rotor's advancing side, called high-speed impulsive noise, and unsteady pressure fluctuations on the rotor blade 
due to interactions with vortices generated by preceding blades, called blade-vortex interaction (BVI) noise. These types of noise are loud and impulsive in nature, which is significant both for military detection and community annoyance.

High speed impulsive noise has been extensively investigated for many years and noise generating mechanisms have been reasonably well understood. The noise is generated by compressibility effect on rotor blades in transonic range and the noise reduction concepts are directly related to reducing or eliminating the compressibility effect of airfoils. The blade tip shape and velocity have been identified as the most important parameters and many practical concepts to control compressibility effects have been successfully used, such as tapering, sweeping, thin blades, or low tip speed. This subject, not part of this review, is further dealt with in Brentner and Farassat, ${ }^{(5)}$ George, ${ }^{(13)}$ Lowson, ${ }^{(24)}$ Marze and Philippe, ${ }^{(26)}$ and Schmitz and Yu. ${ }^{(34)}$

BVI noise has been identified as the most annoying noise source for residential community and the acceptance of rotorcraft hinges on noise reduction capabilities. Noise is generated mainly from unsteady pressure fluctuations on a blade due to BVIs during descent or maneuvering flight conditions. The BVI phenomenon is very complex due to different forms of interactions between a blade and vortices, but near-parallel interactions have been identified as a dominant source for BVI noise. Furthermore. rotor wakes were identified as a major parameter in controlling BVI phenomena and noise. Particularly, tip vortex structures have been extensively investigated in the seventies and eighties with different blade tip shapes, such as Ogee tips, spoilers, or wingtips, ${ }^{(6)}$ but few significant practical design productions have been developed so far, partly due to performance penalties.

Recently, active blade control techniques, such as higher harmonic control ( $\mathrm{HHC}$ ) or individual blade control ( $\mathrm{BC}$ ), were investigated with significant success in reducing BVI noise. The HHC concept excites the blade pitch angle at a fixed frequency through the rotor swashplate collective and cyclic flight inputs. With this, all blades are excited simultaneously at a given frequency through proper phasing of the control inputs, thus the frequency variation is very limited. To increase the control flexibility of input variables, the IBC concept has been developed to independently actuate individual blades in a rotating frame through the rotor hub. The test data indicated that very significant simultaneous reductions in both BVI noise and hub vibrations could be obtained using multiharmonic IBC inputs. To further increase of the control flexibility of input variables, various concepts using distributed smart actuators along the blade span are actively being investigated to control blade tip loading distribution, blade tip deflection, or aerodynamic response on the leading edge during interactions. In this paper, the current active rotor control technology for helicopter BVI noise reduction is reviewed, including the current understanding of how this concept alters the noise generating mechanisms.

\section{BVI NOISE CHARACTERISTICS}

When a rotor operates in descent or maneuvering flight, a rotor blade passes close to trailing tip vortices as shown in Fig. 1, which was taken in the NASA Ames 7- by 10-foot wind tunnel with a model rotor using a smoke visualization. These interactions are plotted in a top view for a typical descent flight condition as shown in Fig. 2, which shows multiple interactions in both the advancing and retreating sides. During these interactions, the blade experiences unsteady pressure fluctuations and generates loud, impulsive noise, called blade-vortex interaction (BVI) noise. A typical noise signature of a four-bladed model rotor in a normal descent flight is shown in Fig. 3, where BVI noise pulses are distinctive and are dominating in the mid-range frequency spectrum. Similar noise signatures are also observed in full-scale flight. ${ }^{(4)}$

An important feature for gaining a better insight into BVI phenomena and associated noise characteristics is the unsteady blade surface pressures. A sample of typical upper surface pressure histories is shown in Fig. 4, where the leading edge pressure transducers clearly identify the aerodynamic BVI phenomena in both the first and fourth quadrants. 


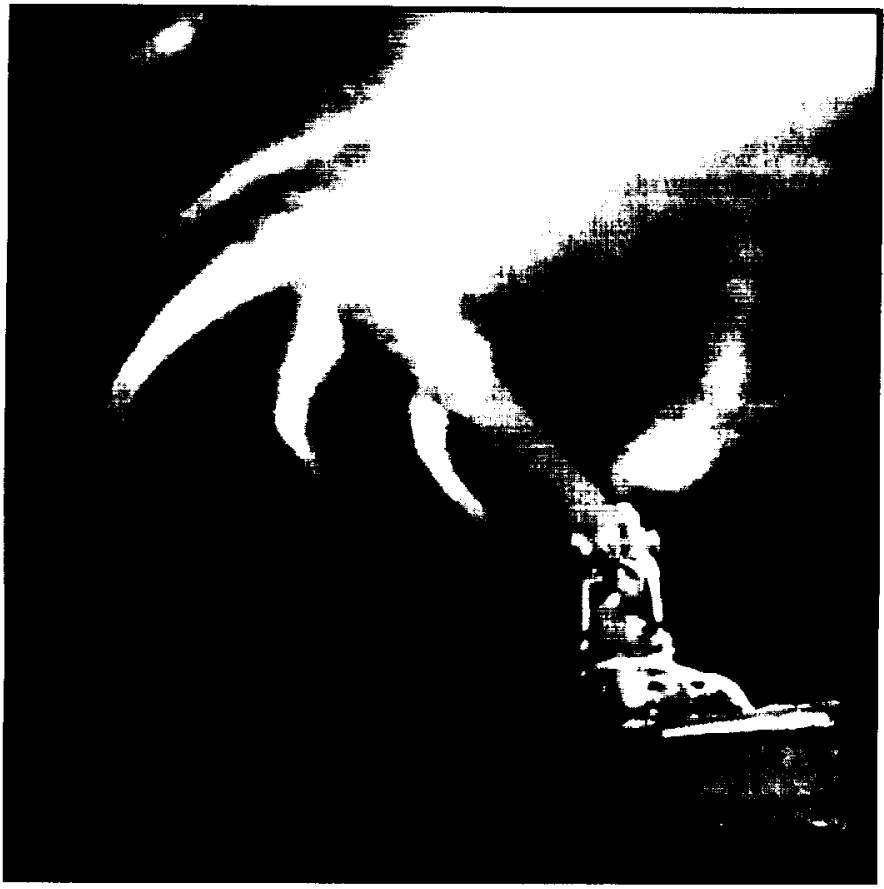

Fig. 1. Blade vortex interactions (BVI) for a descent flight.

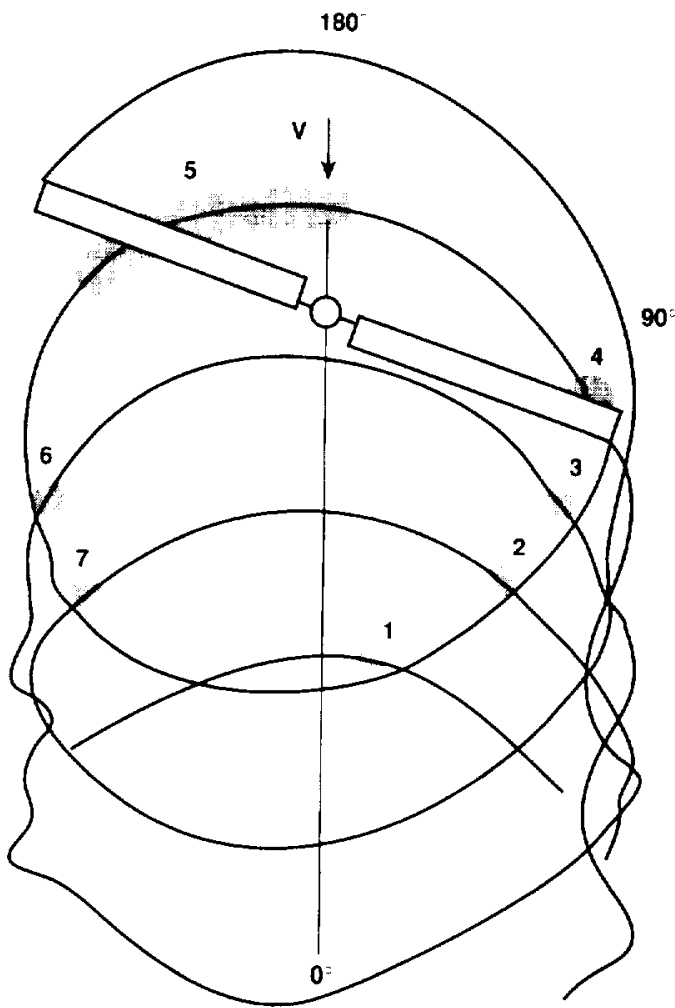

Fig. 2. BVIs during partial-power descent. ${ }^{(46)}$ 

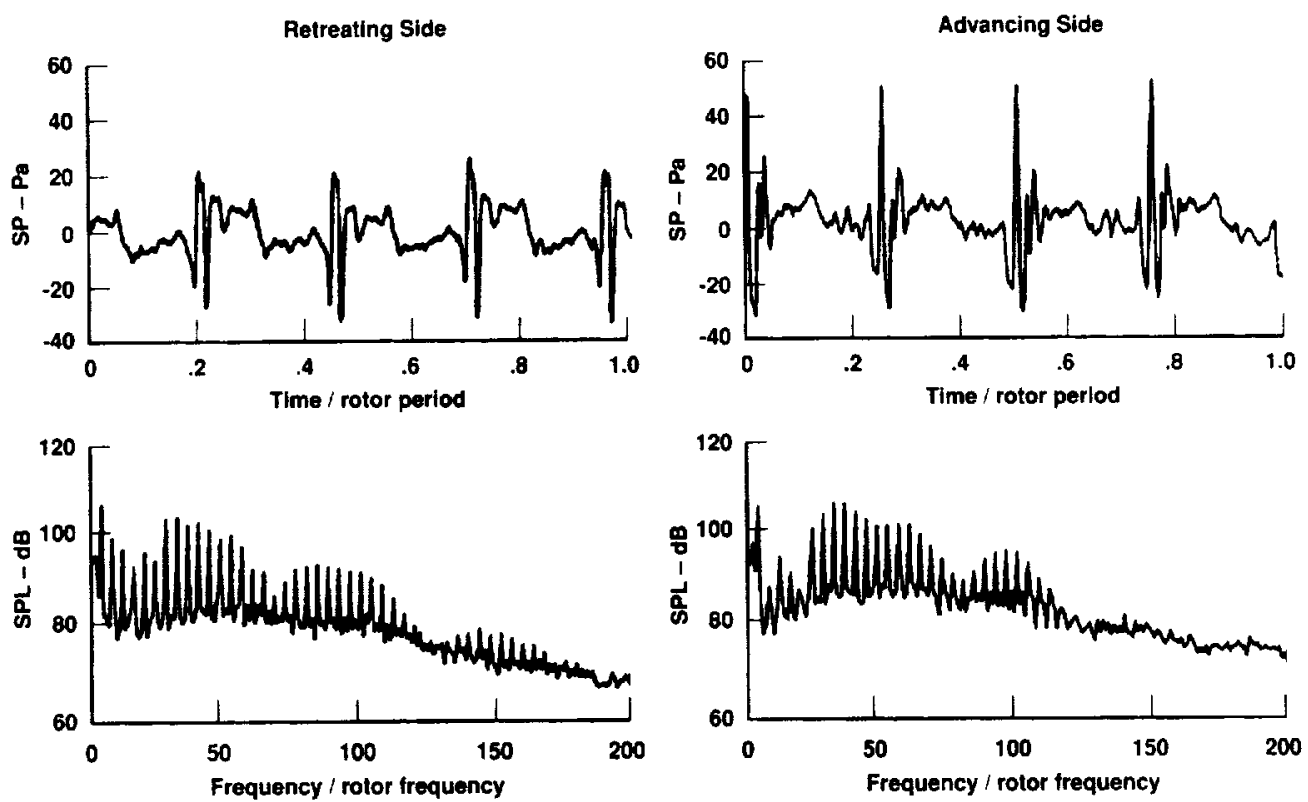

Fig. 3. Typical BVI impulsive noise characteristics. ${ }^{(39)}$

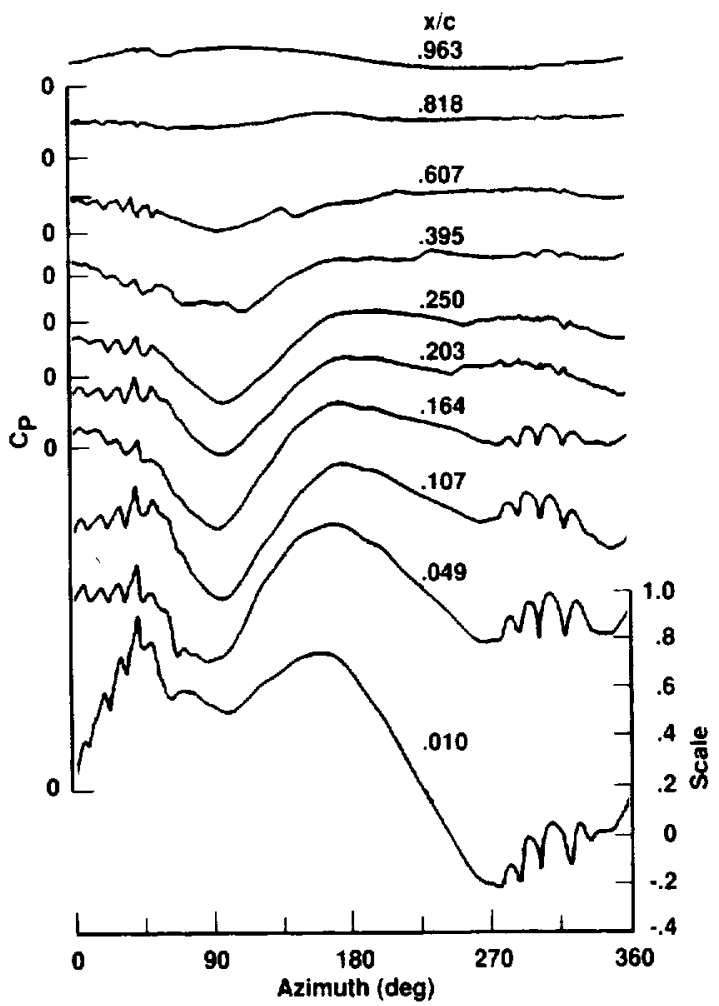

Fig. 4. Blade surface pressure time histories of a model rotor during BVIs. ${ }^{(23)}$

The same features of the leading edge phenomena are observed in full-scale flight tests. ${ }^{(35)}$ One of the important findings from these experimental data is that the BVI phenomena are concentrated near the leading edge of the blade on both the upper and lower surfaces.

Another important feature for understanding the BVI phenomena is the blade section airloads, which can be calculated by integration of measured blade pressures. Figure 5 


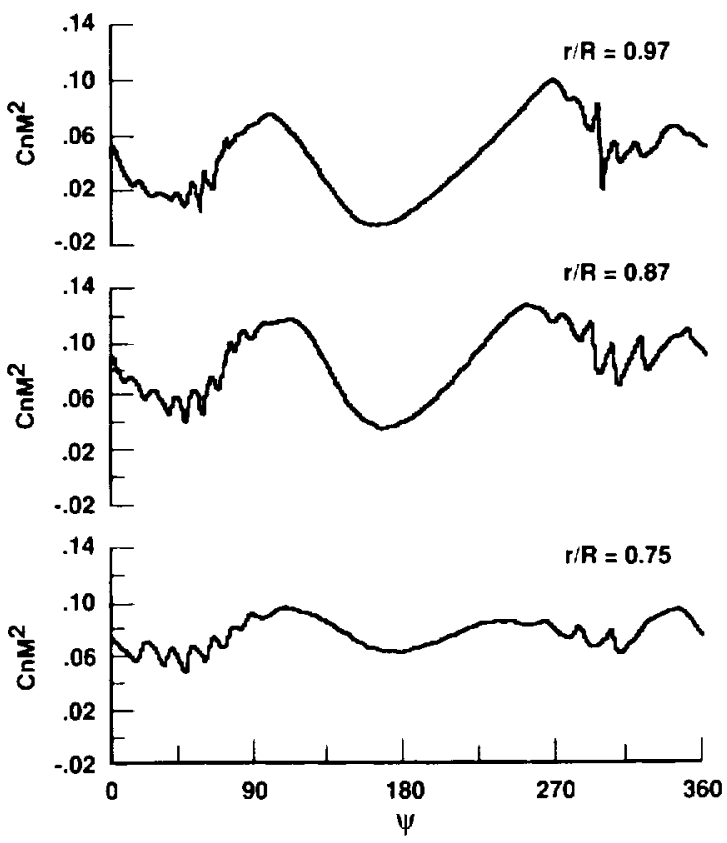

Fig. 5. Blade section airload distributions of a BO- 105 model rotor in a descent flight. ${ }^{(34,}$

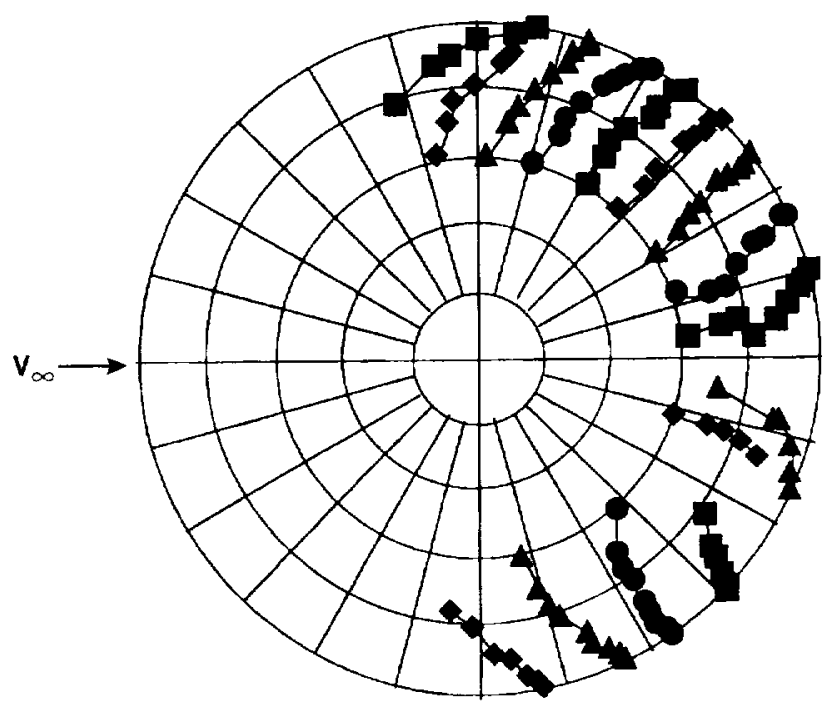

Fig. 6. BVI locations derived from measured leading edge pressures ( $\bullet$ for the vortex blade $1 ; \boldsymbol{\Delta}$ for the vortex blade $2 ; \bullet$ for the vortex blade $3 ;$ and $\boldsymbol{\square}$ for the vortex blade 4$)^{(34)}$

shows typical blade section airload distributions along three spanwise positions, which exhibit multiple BVI interactions in the first and fourth quadrants and determine the rotor wake characteristics, the tip vortex strength, and the BVI geometry. The actual BVI locations in the rotor plane are shown in Fig. 6, obtained from the measured pressures near the leading edge. A number of interactions are seen in the first and fourth quadrants with acoustically important (near parallel interactions) BVIs at about azimuth angles $\psi=50$ and 310 , respectively. With these strong interactions, a typical BVI noise directivity pattern is shown in Fig. 7, where two distinct radiation lobes appear in the advancing and retreating sides. 


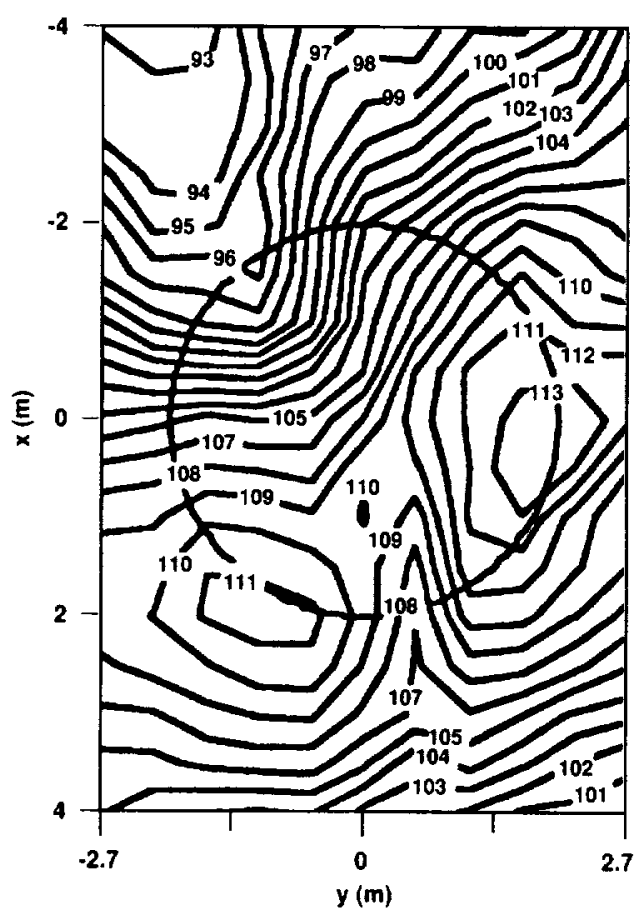

Fig. 7. Mid-frequency BVI noise directivity pattern. ${ }^{(40)}$
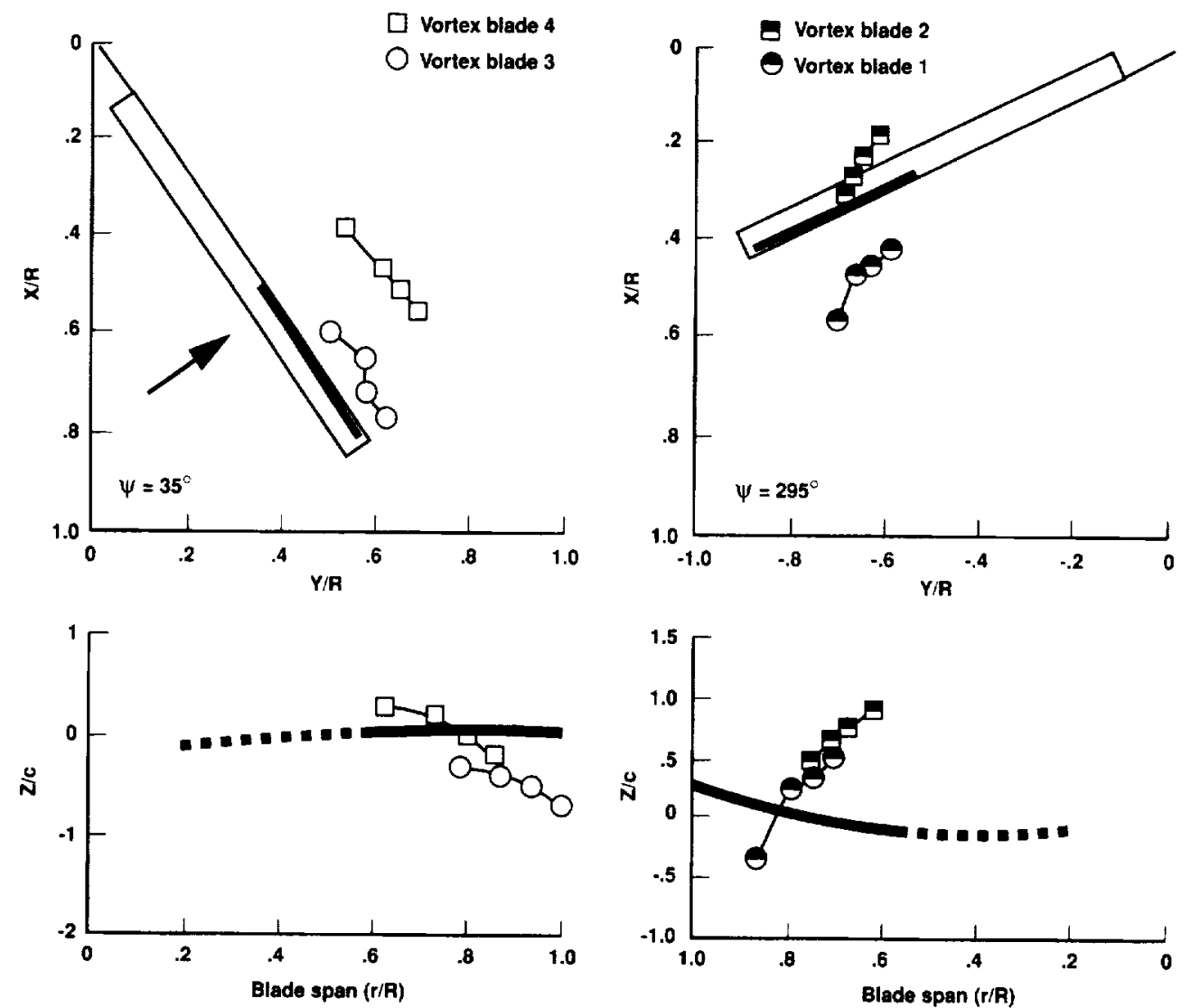

Fig. 8. Tip vortex geometry segments (top views and side views) measured by a laser-light sheet technique. ${ }^{(28)}$ 

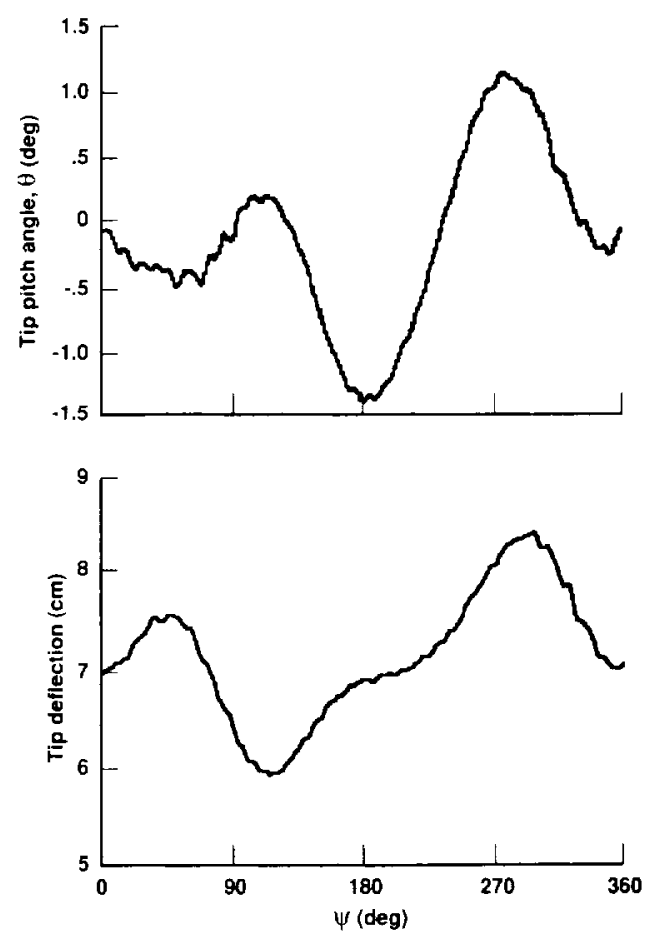

Fig. 9. Blade tip pitch angle and deflection histories along rotor azimuthal angles. ${ }^{(40)}$

Vortex geometry segments of two tip vortices, both significant to BVI, on both the advancing and retreating sides are shown in Fig. 8 . These were obtained from measuring the vortex core centers in space of at least four discrete sections along the vortex of interest. The data were measured for the blade azimuthal positions of $\psi=35$ and 295 , respectively. These figures illustrate both the top and side views of the wake geometry segments for the advancing and retreating sides. From the side view of this figure, the blade vortex miss distance is very small, indicating intense BVI noise generations. In order to gain a better insight of the blade--vortex miss distance, the time histories of blade tip deflection and pitch angle are derived from the strain gauge measurements as shown in Fig. 9. Using the blade flapping and torsional characteristics, the blade tip deflection history along the azimuthal angle can be modified to control the blade-vortex miss distance and eventually the BVI noise by utilizing active blade control concepts such as HHC or IBC. However, rotor noise reduction is extremely complicated due to the fact that rotor blades are not only the main source of noise, but also predominantly the source of rotorcraft performance and control.

\section{ACTIVE ROTOR CONTROL CONCEPTS}

When a helicopter is in forward flight, the blades encounter nonsymmetric velocities on the left- and right-hand sides of the aircraft. The velocity differences are compounded by the downwash velocities of a helical trailing vortex system. These effects produce such aerodynamic problems on the rotor blade as blade stall and drag divergence. These aerodynamic phenomena affect the helicopter's flight characteristics and performance and limits its operational flight envelope.

Helicopters are conventionally controlled via a swashplate sliding up and down the rotor shaft and tilting relative to it. This system is very successful in producing monocyclic (i.e. one cycle per rotor revolution) blade feathering angles, allowing the pilot to control the helicopter's position and attitude. However, this control system is not capable of preventing blades from entering adverse flow conditions during steady or maneuvering flight. The 
potential solution of this problem is to sense rotor behavior and act through additional or new control inputs to eliminate or reduce the detrimental characteristics; the answer is active rotor control. ${ }^{(18)}$

Several types of rotor active control concepts are shown in Fig. 10, from full blade feathering through direct lift flap and controllable twist to different kinds of circulation

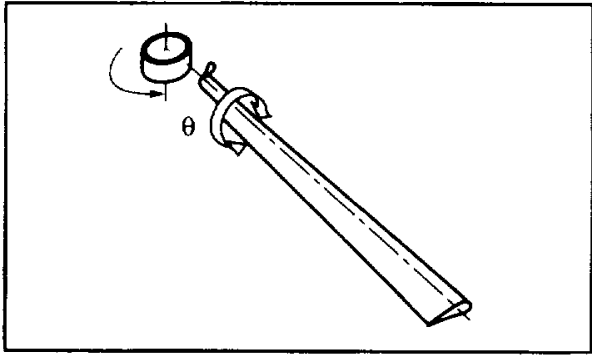

(a) Full blade feathering

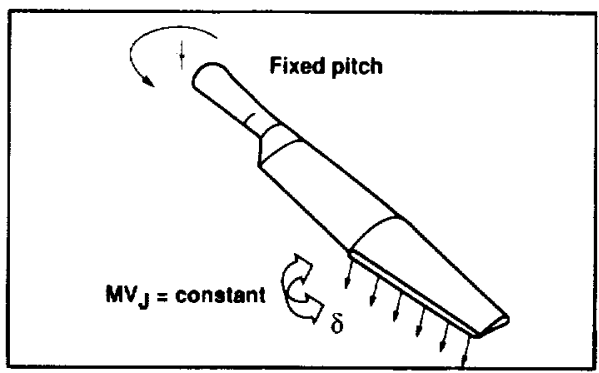

(c) Propulaive jet flap

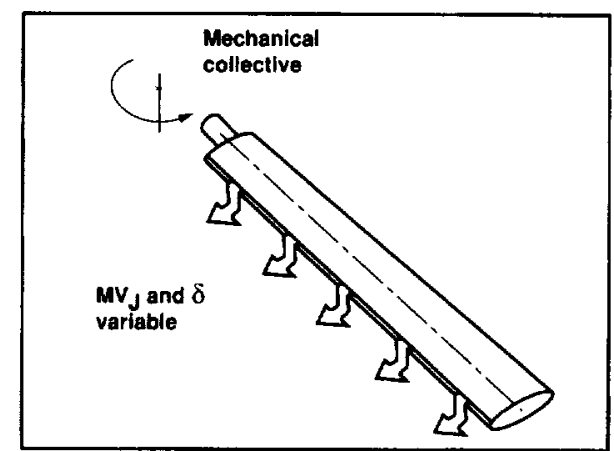

(e) Circulation control

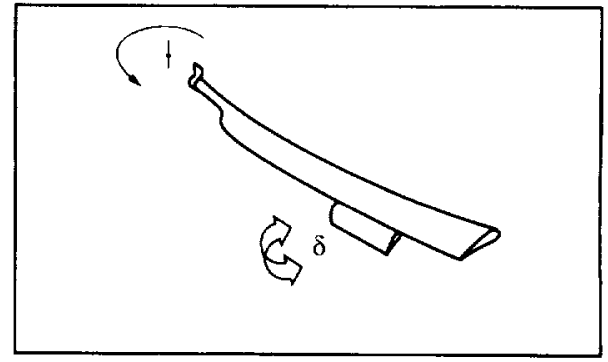

(b) Controllable twist

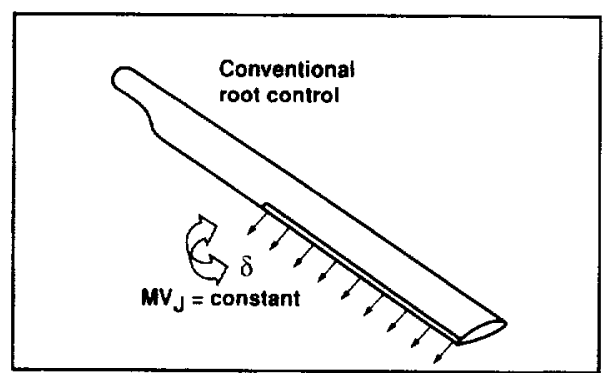

(d) Nonpropulsive Jet flap

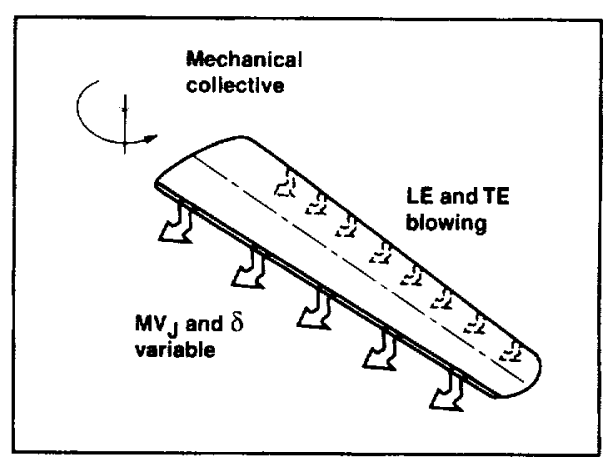

(f) $x$-Wing

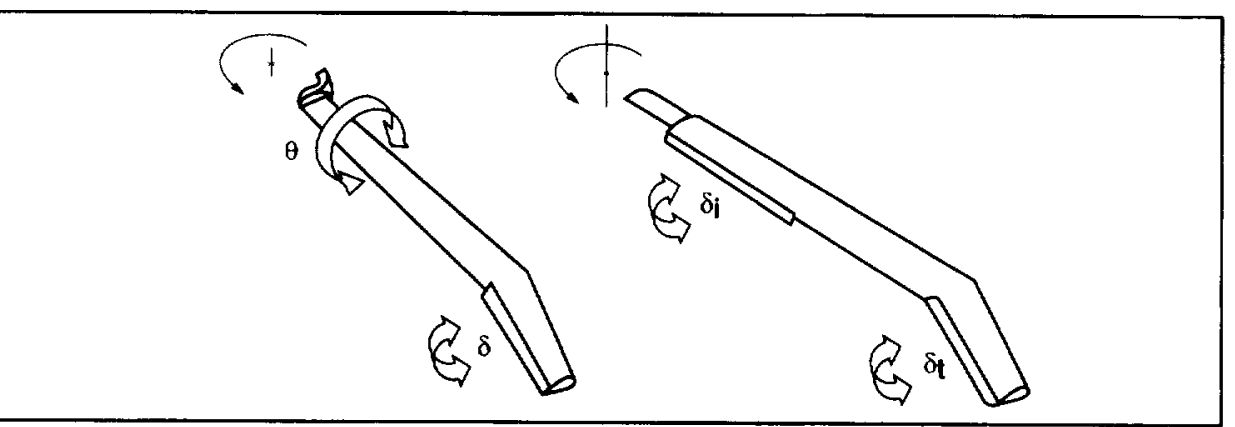

(g) Advanced center blades

Fig. 10. Rotor types with active control concepts. ${ }^{\{27}$ 
control. Extensive theoretical and experimental studies have been conducted in order to investigate these concepts. The high potential benefits of these concepts are:(1) reduction of vibration, noise, control loads, blade stresses and power input; (2) avoidance of stall, compressibility effects, gust and disturbance responses, and vortex interactions; and (3) elimination of rotor instabilities.

\subsection{BLADE ROOT CONTROL CONCEPTS: HHC AND IBC}

During the last two decades a large number of investigations were concerned with active blade control systems utilizing full blade feathering or active blade root control: the higher harmonic control (HHC) system, which is characterized by the implementation of the actuators below the swashplate, and the individual blade control (IBC) system, in which each blade is controlled individually by pitch link actuators in the rotating system (Fig. 11).

The original intention in developing and applying $\mathrm{HHC}$ for rotors was to improve helicopter ride quality (i.e. to reduce the considerable vibration level). Numerous analytical and experimental studies demonstrated that $\mathrm{HHC}$ is capable of reducing the vibration level up to $90 \%$ and to provide 'jet smooth' ride qualities for the helicopter.

The rotor induced vibration of a helicopter originates from the unsteady aerodynamic forces acting on the rotor blades, which are caused by reverse and radial flow as well as shock and stall effects and are clearly associated with discrete rotor azimuth positions. Consequently, the aerodynamic blade loads are periodic with the corresponding frequency spectra mainly consisting of rotor harmonics. These spectra reveal strong amplitudes in the low-frequency range (Fig. 12) which, due to the limited blade stiffness, excite the rotor blades to oscillate in flapwise and lead-lag direction. These deflections generate inertia and elastic forces, in combination with the aerodynamic excitations forming the resulting blade loads (Fig. 13). Summing up these blade loads to yield the forces acting in the fixed system, the individual harmonics substantially cancel each other. The remaining loads are integral multiples of the blade passage frequency

$$
P_{n}=(h+n) P
$$

where $n$ is an integer number, $b$ is the number of blades, and $P$ is the rotor rotational frequency or period.
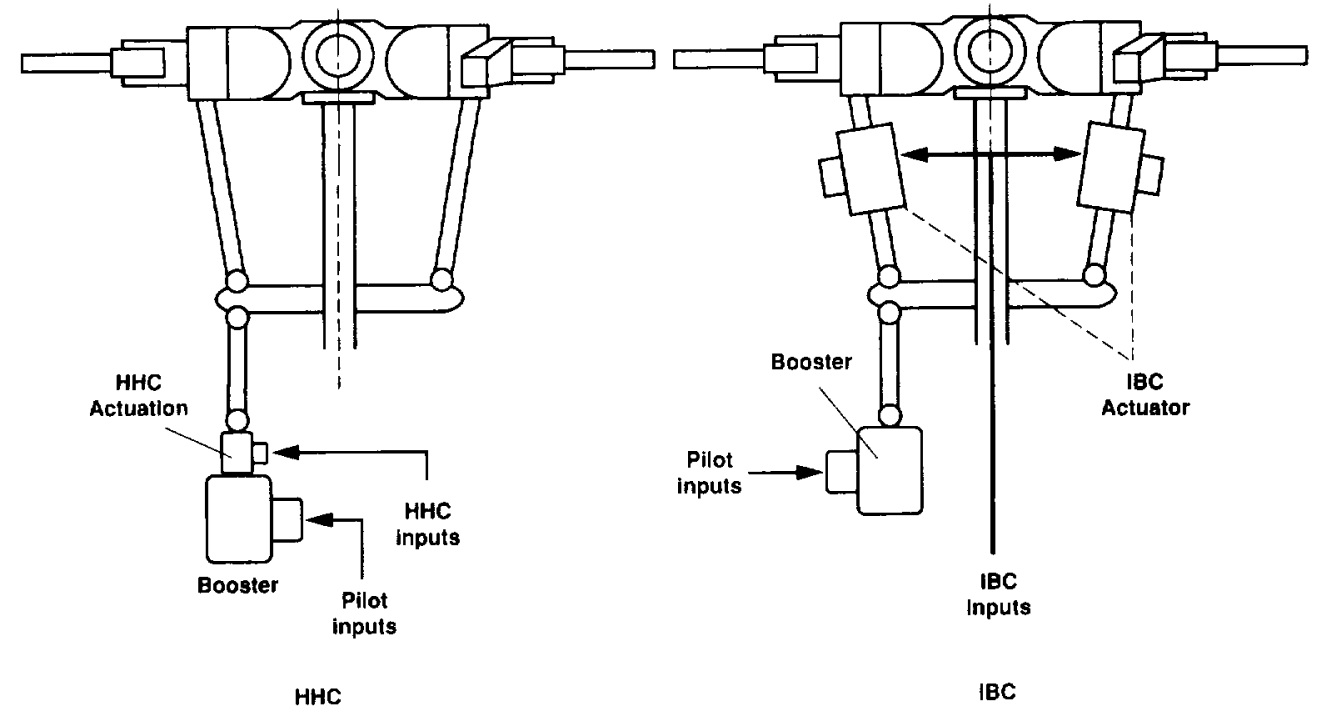

Fig. 11. Active blade root control concepts (HHC/IBC).201) 


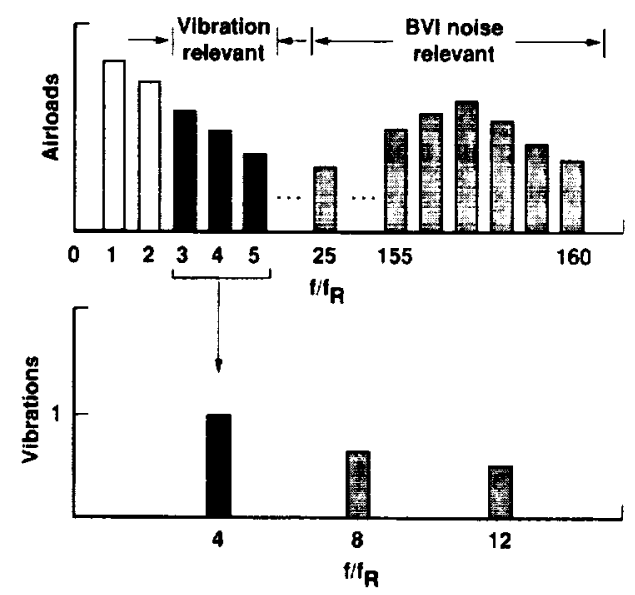

Fig. 12. Airload and vibration characteristics. ${ }^{(20)}$

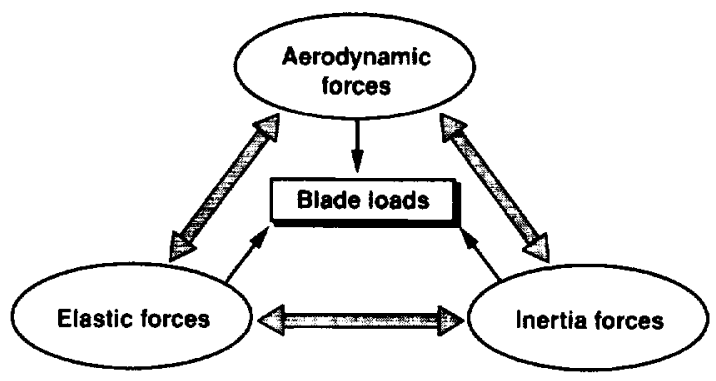

Fig. 13. Resulting blade loads. ${ }^{(20)}$

From these harmonics, the lowest one not only is associated with the strongest amplitude (Fig. 12), but also represents the most annoying part. It is caused by blade loads in the frequencies of $(b-1) P, b P$, and $(b+1) P$ harmonics, which can very well be affected by a blade pitch angle exactly at these frequencies. If the corresponding amplitude and phase shift values are properly adjusted, the blade oscillations can be controlled in a way that leads to a reduction of the corresponding hub loads and therefore to a minimization of the vibrations in the fuselage.

A potential benefit of the HHC concept for BVI noise reduction was suggested in the late 1980 s based on analytical investigations. ${ }^{(15)}$ The impulsive noise is due to BVIs around $\psi=50$ and $310^{\circ}$, respectively, with the vortices generated within the proximity of $\psi=130$ and $230^{\circ}$. In a first step, BVI noise can be assumed to be proportional to the inverse blade-vortex miss distance squared and the vortex strength parameters which, like the low-frequency blade loads, can very well be affected by means of a high-frequency blade pitch angle. If the amplitude and the phase shift of this blade pitch angle are adjusted in a way that leads to a decreased blade angle of attack at the rotor azimuth positions where the interacting vortices are generated, the vortex strength and BVI noise emissions can be expected to be reduced.

However, this decreased blade angle of attack may cause blade oscillations which lead to an increase of the vibration level in the fuselage. In this respect a blade pitch angle of the frequency $(b-2) P$ becomes important because it has the potential to reduce the BVI noise emissions while affecting the vibrations through the usually small interharmonic couplings.

Contrary to blade pitch angles of the frequencies $(b-1) P, b P$, and $(b+1) P$, however, a blade pitch angle of the frequency $(b-2) P$ cannot be realized by implementing the actuators below the swashplate, but requires actuators in the rotating frame (Fig. 11). 
Substituting the conventional pitch links, these actuators make it possible to control each blade individually, while the implementation of the actuators below the swashplate affects all blades simultaneously, and due to the swashplate kinematics this concept is, in addition. limited to blade pitch angles of the frequencies $(h-1) P, b P$, and $(b+1) P$.

Most of the work in the field of active rotor control has centered on control schemes using actuators in the fixed system. The HHC method was thoroughly investigated and tested through analysis, wind tunnel and flight testing. Rotors with actuators in the rotating system to individually control each rotor blade, the IBC method, have been investigated in theoretical simulations, wind tunnel experiments and, to a limited extent, flight tests.

The first flight test program using a higher harmonic blade feathering control technique was conducted to control vibration of a four-bladed helicopter rotor by the McDonnell Douglas Helicopter Systems (MDHS). The program consisted of three phases. In its first phase, the MDHS OH-6A helicopter underwent numerous changes to incorporate the HHC system. The actuator installation is shown schematically in Fig. 14 for the four-bladed hub. The system superimposed fourth harmonic inputs upon the stationary swashplate. These inputs were transformed into blade feathering angles with the frequencies $3 P, 4 P$ and $5 P$ in the rotating system. In the second phase, the open-loop or manual controlled flight tests were performed. In the final phase, the closed-loop or computer controlled system was tested in 1983 and 1984 . With the HHC system engaged, the $4 P$ pilot seat vibration levels were significantly lower than the baseline helicopter (Fig. 15), and furthermore the system did not adversely affect blade loads or helicopter performance.

Aerospatiale Helicopter Division (now ECF) launched a research flight program in 1980 to test an active vibration control system based on $\mathrm{HHC}^{(32)}$ The system was installed on the experimental helicopter SA 349, derived from the SA 342 Gazelle. In Fig. 16 the main elements of the higher harmonic control system on the SA 349 helicopter are shown; these include the swashplate control system ( $3 P$ in the nonrotating system) generating control angles at the frequencies $2 P, 3 P$ and $4 P$ in the rotating system with a maximum $\mathrm{HHC}$ amplitude of 1 . The HHC self-adaptive system is shown schematically in Fig. 17. Three control algorithms were developed and flight-tested, demonstrating a fast adaptive control capability to changing flight conditions. The system was tested in the closed-loop mode throughout the flight envelope of a conventional helicopter. In cruise flight at $250 \mathrm{~km} / \mathrm{h}$

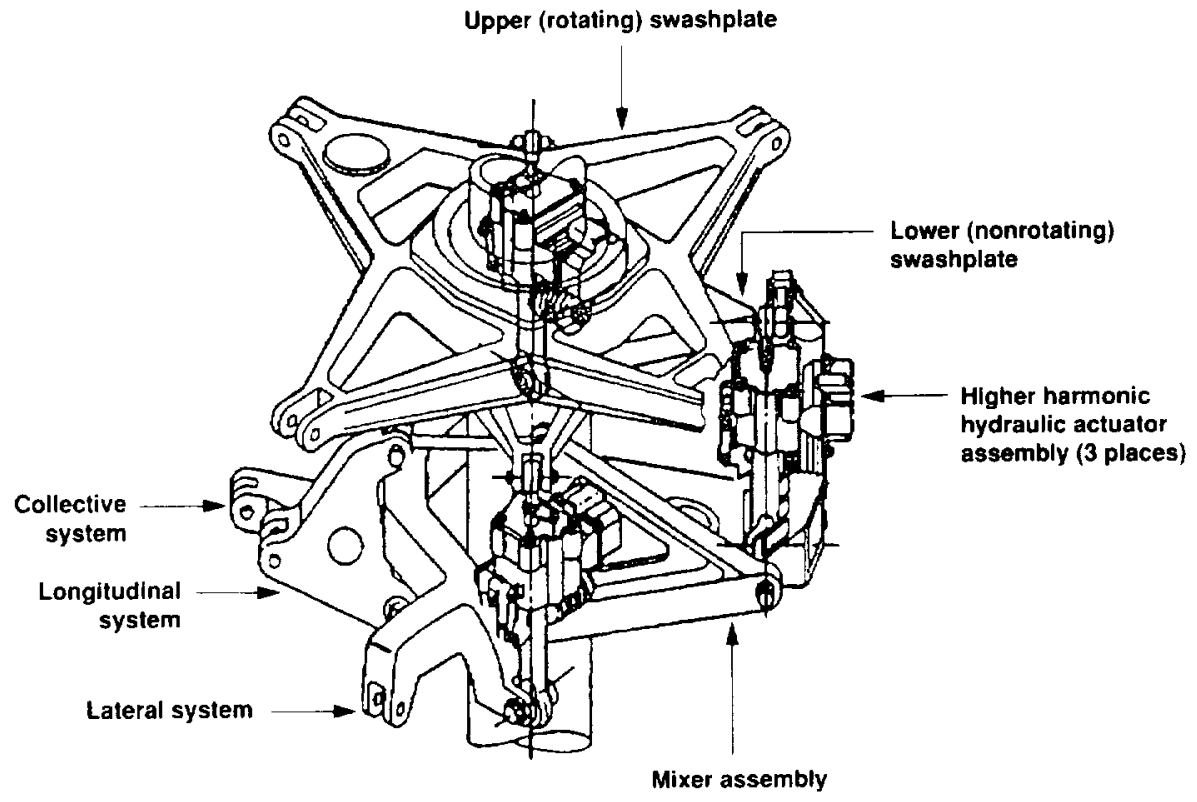

Fig. 14. HHC actuator installation schematic on OH-6A helicopter. ${ }^{(43)}$ 


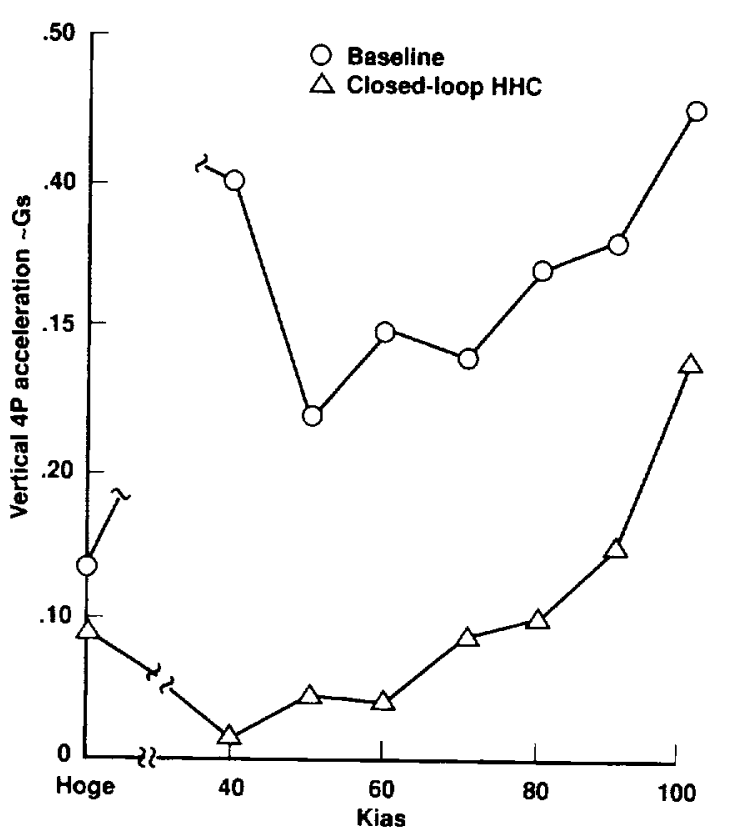

Fig. 15. Vertical pilot seat accelerations $(4 P)$ us airspeed. ${ }^{(43)}$

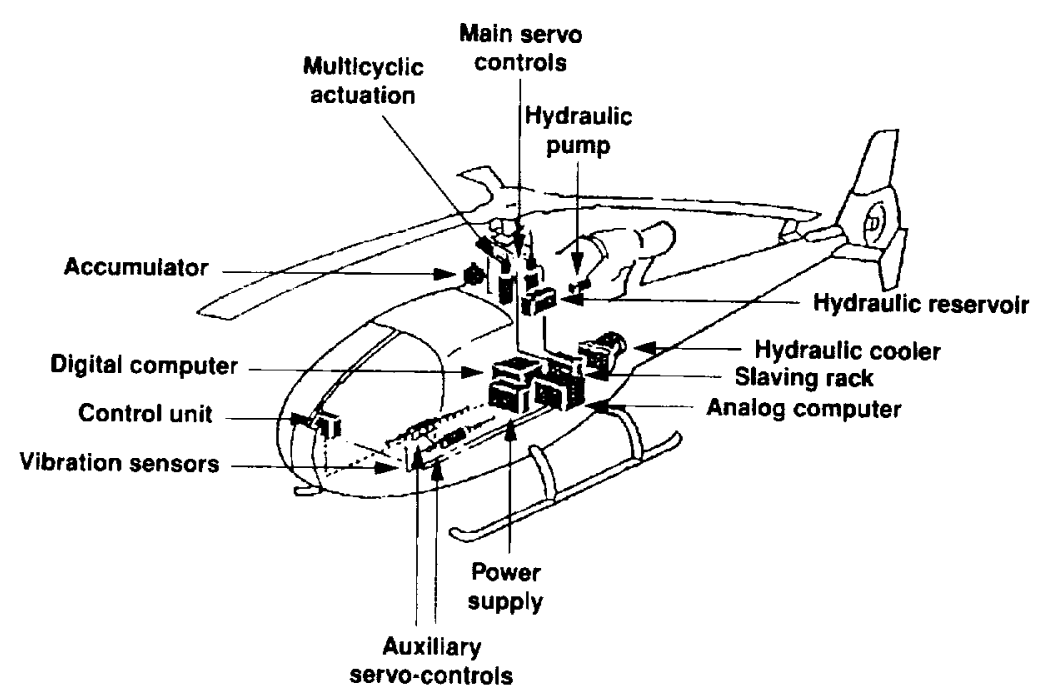

Fig. 16. HIHC system on SA $349 !^{1321}$

the mean reduction in vibration level of $80 \%$ was obtained in the cabin with local reductions of $95 \%$ at certain locations. Figure 18 presents flight test results obtained in 1985 from the HHC equipped helicopter in comparison with the basic helicopter and a helicopter equipped with a passive-type vibration reduction system.

Flight tests of an open-loop higher harmonic vibration control system were conducted by Sikorsky Aircraft on an S-76A helicopter during $1985 .^{(48)}$ The HHC system installation was designed to minimize modifications to the standard aircraft configuration. HHC inputs were generated by three hydraulic actuators installed in the nonrotating control system, providing blade feathering at frequencies of $3 P .4 P$ and $5 P$ in the rotating system. The flight tests included evaluations of $\mathrm{HHC}$ inputs in the longitudinal, lateral and collective axes at varying amplitudes and phases. Significant vibration reductions throughout the aircraft 


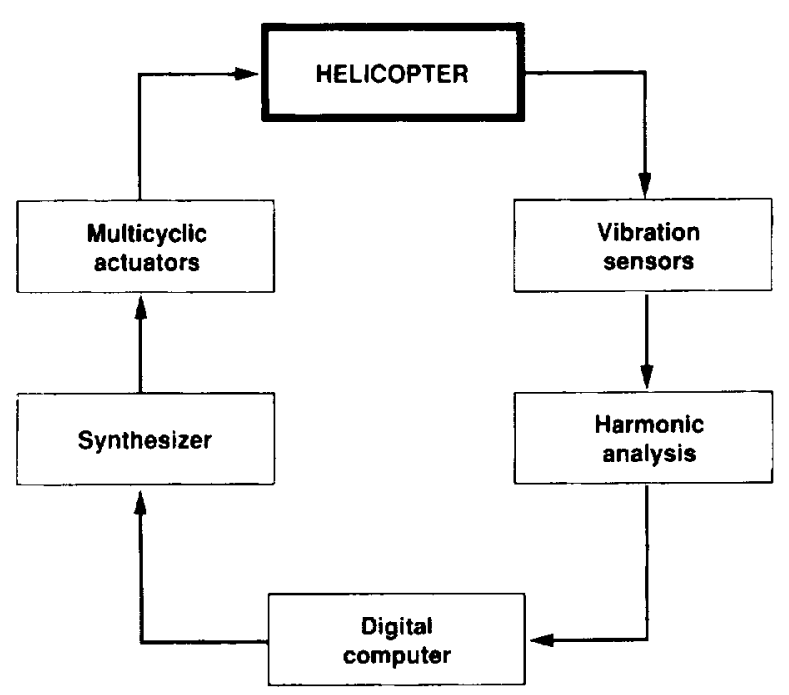

Fig. 17. HHC self-adaptive system..$^{32 !}$

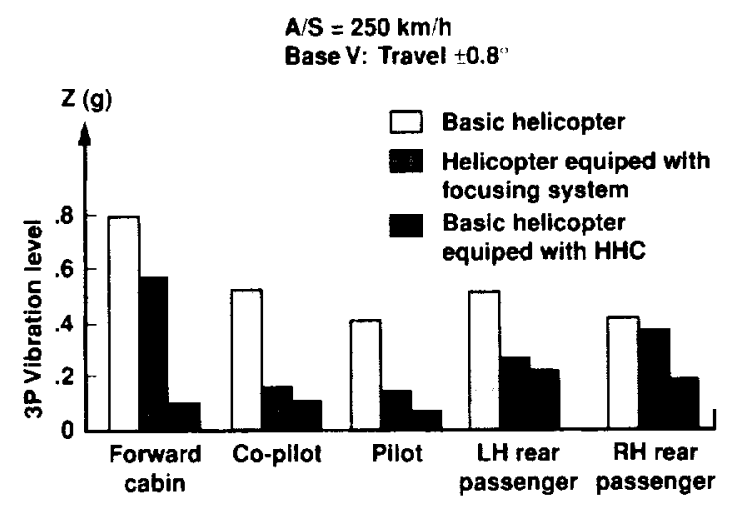

Fig. 18. HHC flight test results. ${ }^{(32)}$

were demonstrated at forward speeds up to 150 knots (Fig. 19). The capability of HHC to reduce vibrations was also demonstrated at varying rotor speeds and during maneuvers. Structural data obtained during testing showed a general increase in control system loads during HHC operation. No observable degradation of aircraft performance was noted during limited performance testing with the HHC system operational.

The HHC systems described above were investigated with the main objective of reducing the vibration level at certain locations of the helicopter. Subsequently, additional potential that HHC may also be effective for BVI noise reduction was pursued and tested. For the first time, this benefit was demonstrated in 1988 by wind tunnel tests of DLR in the DNW ${ }^{(37)}$ and of NASA in Langley Research Center's TDT. ${ }^{(8)}$ In Fig. 20 the HHC effect on BVI noise generation of a BO-105 model rotor is shown for different control modes with control frequencies of $3 P, 4 P$ and $5 P$. By comparing these results to corresponding baseline conditions (without $\mathrm{HHC}$ ), significant noise reductions of 4 or $5 \mathrm{~dB}$ were found for low-speed descent conditions, where helicopter BVI noise is most intense. During the tests. it was observed that the vibration levels were unexpectedly increased in low noise control settings, and no control setting was found for reduction of both vibration and noise at the same time. In the following systematic test programs, ${ }^{(38)}$ it was confirmed that the rotor vibratory loads increase and may even exceed the baseline level without $\mathrm{HHC}$ at certain 

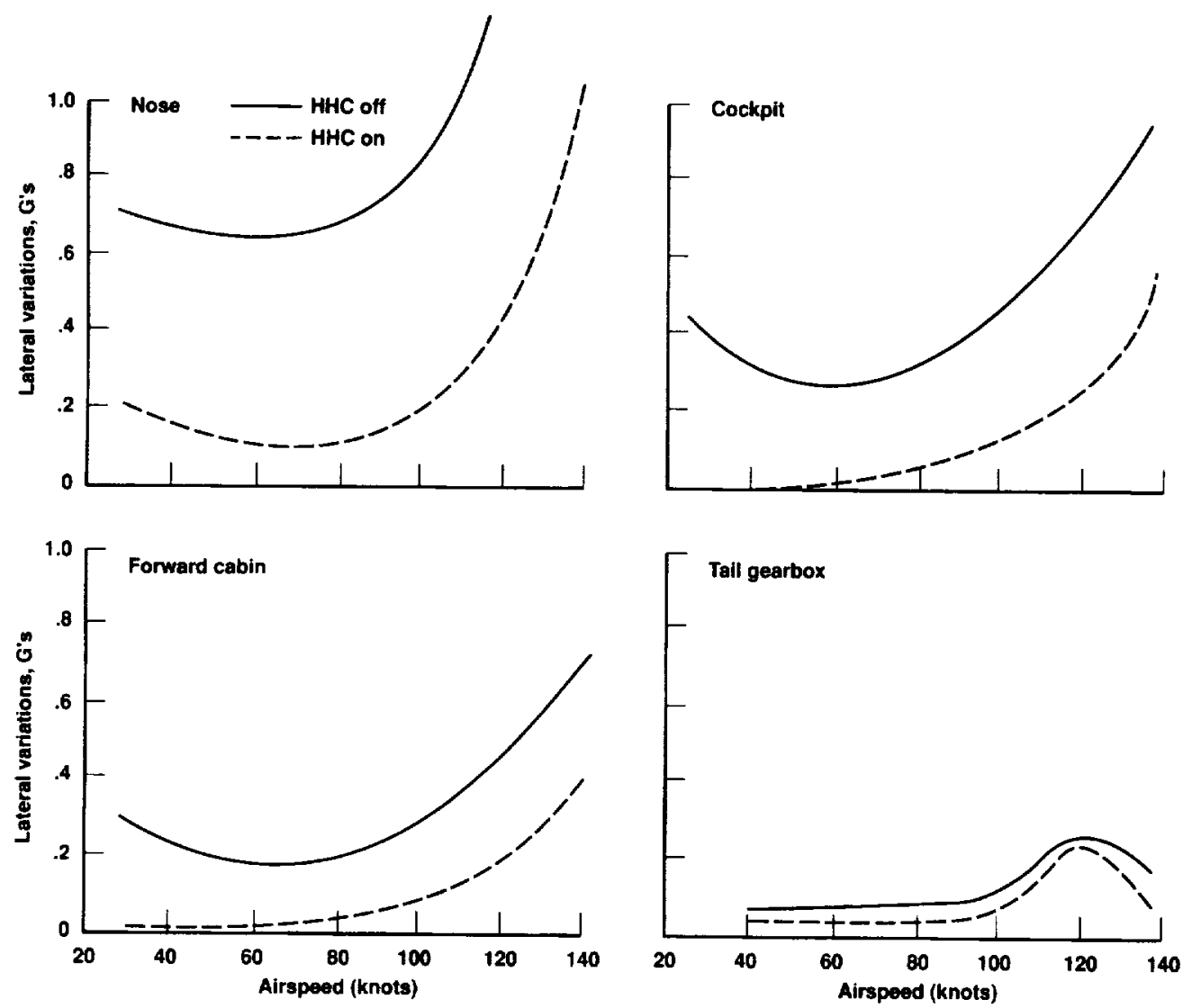

Fig. 19. Effect of HHC on aircraft vertical vibration. ${ }^{(48)}$

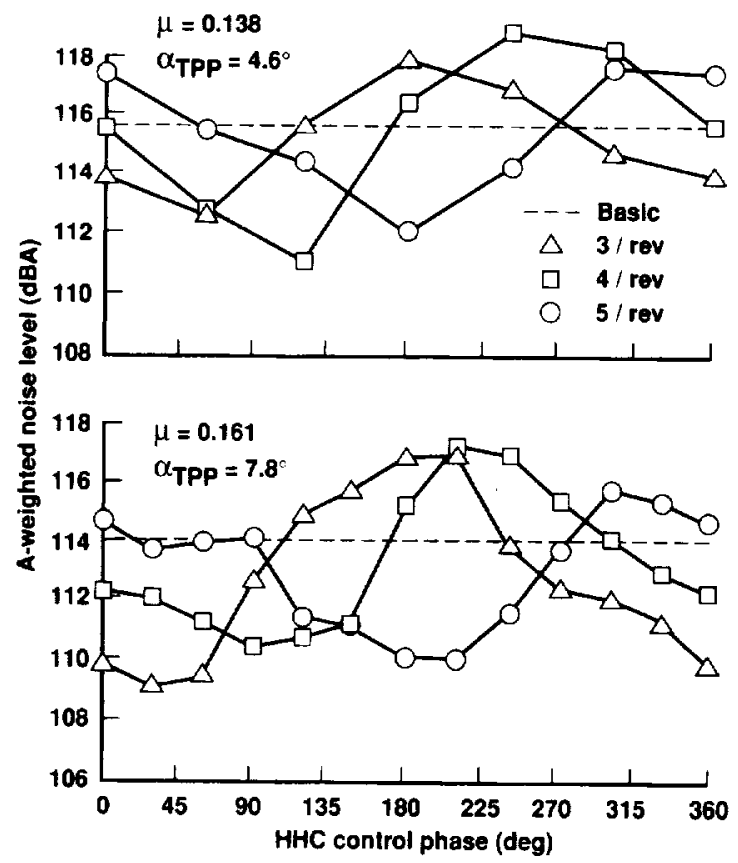

Fig. 20. HHC effect on BVI noise generation for different control modes. ${ }^{(37)}$ 
control settings that are most beneficial for BVI noise reduction. This effect was not well understood at that time.

With the objective of simultaneously reducing the vibration level, the BVI noise, and the power required, and in addition, to avoid rotor instabilities, an IBC system was developed at ZF Luftfahrttechnik (ZFL) in Germany. ${ }^{(33)}$ The system was implemented by replacing the rotating control rods of a BO- 105 helicopter with hydraulic pitch-link actuators (Fig. 21). The actuators operate hydraulically and are controlled by servovalves. In case of hydraulic pressure loss, the actuators are locked by springs in a definite position and act like conventional pitchlinks. A hydraulic slipring located below the gearbox is used in order to transfer the hydraulic power from the nonrotating power supply system through the shaft to the hub.

The first flight tests of a four-bladed rotor controlled by an IBC system took place in 1990 and 1991 in cooperation between ZFL and ECD. In order to reduce the number of free control parameters, the IBC system was first tested in a harmonic mode with open-loop control inputs with $2 P$ to $5 P$ frequencies. For safety reasons, the control authority, the flight velocity, and the load factor were limited during the flight tests. The maximum blade pitch angle amplitude was 0.4 and the flight velocity was between 61 and 113 knots. The results of these flight tests were promising, but due to the amplitude limitations of the flightworthy control system it was not possible to demonstrate the full capability of the IBC technology.

Subsequently, two full-scale wind tunnel tests were conducted to evaluate the full potential of IBC to improve rotor performance, to reduce BVI noise, and to alleviate helicopter vibrations, under the cooperation between NASA, the U.S. Army, ZFL, ECD and DLR. The IBC servo-actuators and control system were designed and manufactured by ZFL and installed on the Rotor Test Apparatus (RTA) equipped with a full-scale BO-105 rotor system in the 40- by 80 -foot wind tunnel at Ames Research Center. ${ }^{(17)}$ In Fig. 22 the IBC actuators and the BO- 105 rotor system are shown on the RTA. The data generated in the two test programs in 1993 and 1994 indicated that significant simultaneous reductions of both BVI noise and hub vibrations could be achieved using multiharmonic IBC inputs. The data also showed that performance improvements of up to $7 \%$ could be obtained using $2 P$ inputs at high-speed forward flight conditions.

Figure 23 shows simultaneous noise and vibration reduction using different control amplitudes at $2 P$ frequency and at a phase angle of $60^{\circ}$. BVI noise reductions of up to $10 \mathrm{~dB}$ locally were obtained in front of the rotor on the advancing side, with the average reduction being in the range of about $4-8 \mathrm{~dB}$. The best reduction achieved in $4 P$ hub loads was on the order of $90 \%$ with simultaneous noise reductions as noted above.

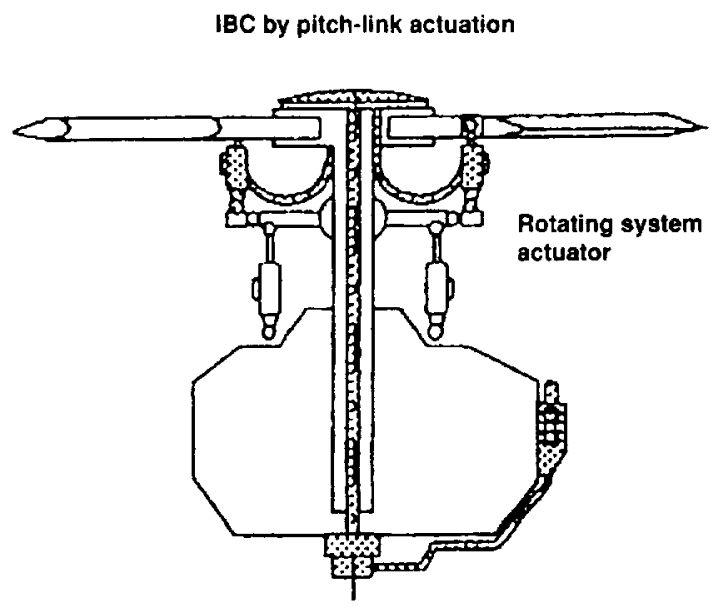

Fig. 21. IBC hydraulic pitch-link actuators. ${ }^{(33)}$ 

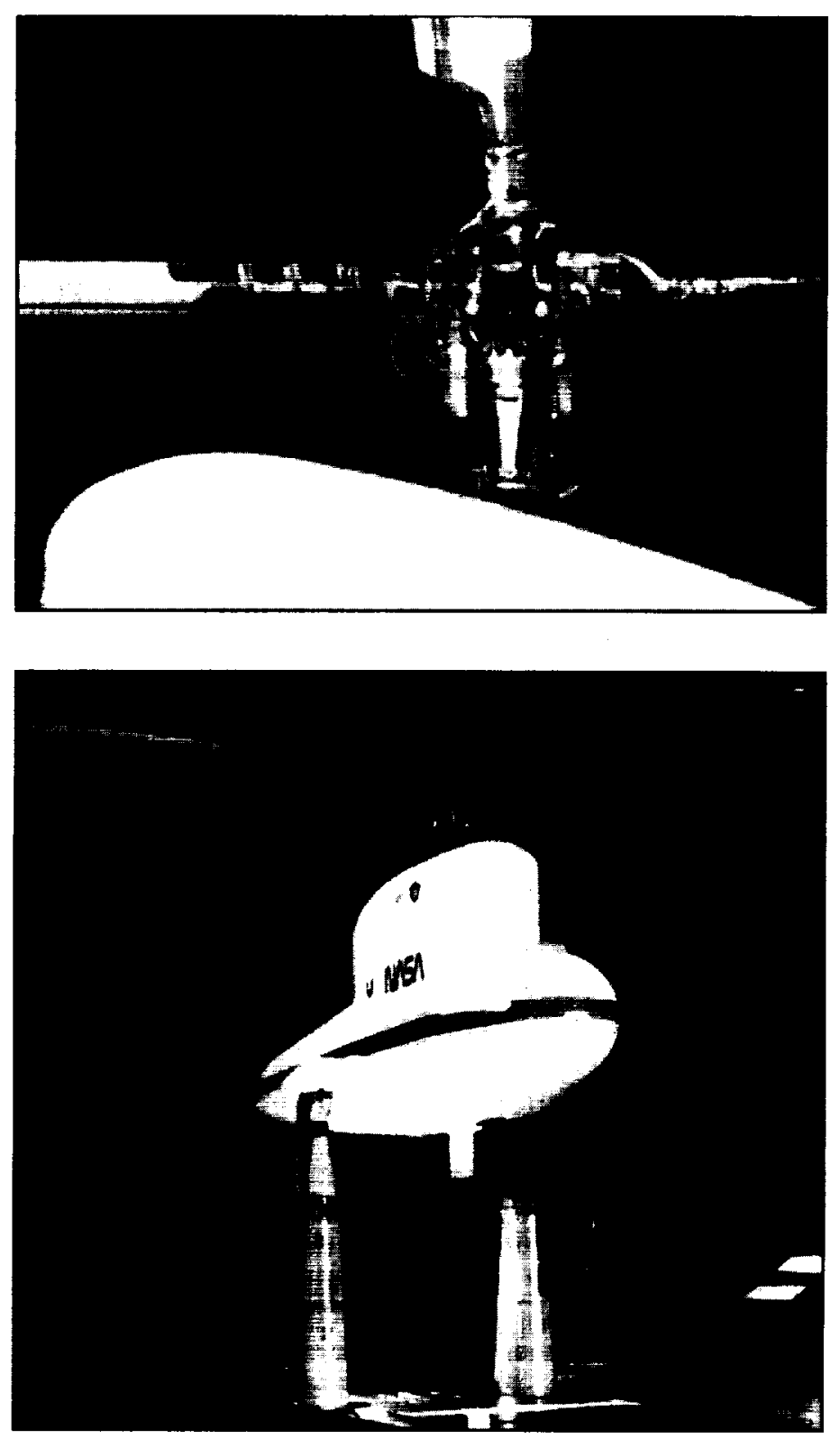

Fig. 22. BO-105 rotor system in the $40 \times 80$ wind tunnel. ${ }^{117}$

\subsection{CONTROLLER DESIGN}

For the performance of the whole active control system, in addition to the hardware for rotor control, the closed-loop controller design is of utmost importance. Numerous approaches have been proposed and some have been implemented and tested. Table 1 provides a selection of controllers proposed or applied for vibration reduction using HHC. It shows that most of the investigated controllers are adaptive ones in the frequency domain and can provide vibration reduction of about $80-90 \%$.

The controller in the frequency domain assumes a steady state operating condition and a quasi-steady dynamic rotor behavior. This means that the rotor response and the control inputs are periodic, and the control law can be expressed in terms of the Fourier coefficients 


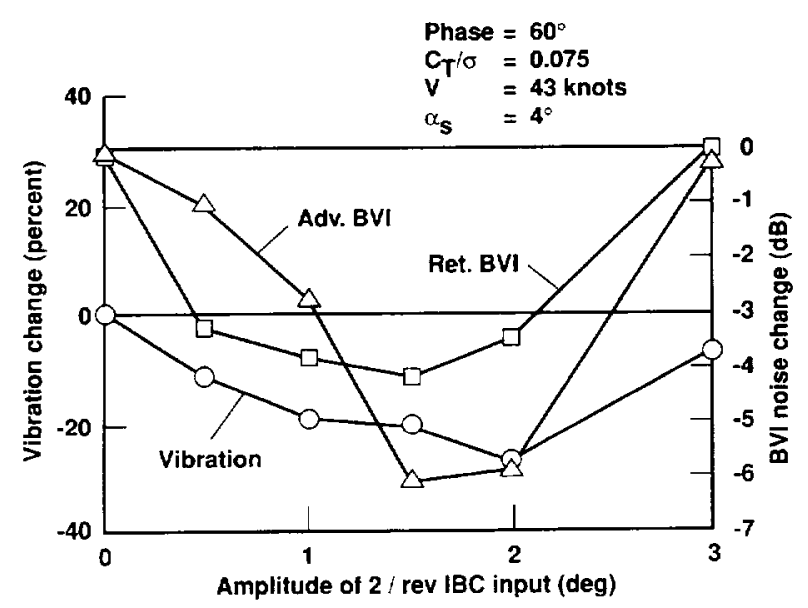

Fig. 23. Correlation of BVI noise and vibrations for control inputs with $2 P$ frequency. ${ }^{117}$

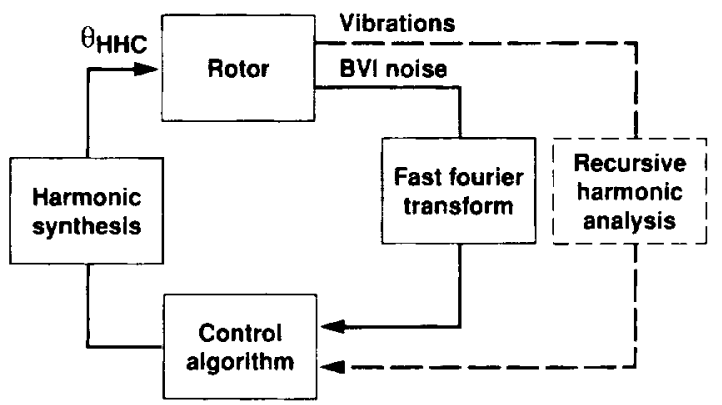

Fig. 24. Block diagram of a frequency domain HHC controller for vibration and noise reduction. (21)

of the rotor response and the resulting control inputs. Consequently, a harmonic analysis and a harmonic synthesis are required for implementing the frequency domain controller. Figure 24 shows a block diagram of a closed-loop controller in the frequency domain designed for BVI noise and vibration reduction using HHC inputs.

In the case of a time domain control, the operating condition of the rotor is not necessarily assumed to be steady and, therefore, the realistic representation of the dynamic response behavior of the rotor is essential for the controller. In addition to the other benefits, with a time domain controller the stability of the system can be improved by an appropriate feedback gain. This system stabilization is not only beneficial for improving the damping characteristics of the rotor, but also it can be used for high frequency disturbance rejection control tasks. ${ }^{(47)}$

Up to now, a frequency domain control was preferred for active rotor control applications due to the complex dynamic behavior of the rotor. For time domain controllers sophisticated methods for rotor modeling and controller design are required. As known, the structural blade and rotor response due to a single-harmonic input contains also harmonics other than the original excitation frequency. The origin of these inter-harmonic couplings is partly due to dynamic pressure variations which increase with the helicopter advance ratio. This modulates the single harmonic angle-of-attack variation in multiharmonic lift variations. In addition, the circulation variations, introduced by the active control, produce inflow and angle-of-attack variations. Modern rotor analysis tools and advanced controller design methods are able to cope with these complex rotor characteristics. An HHC time 


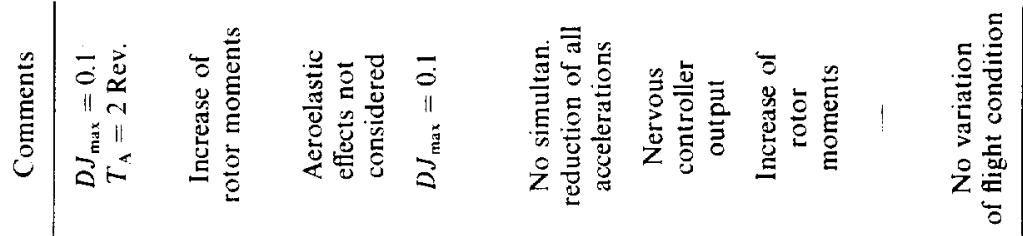

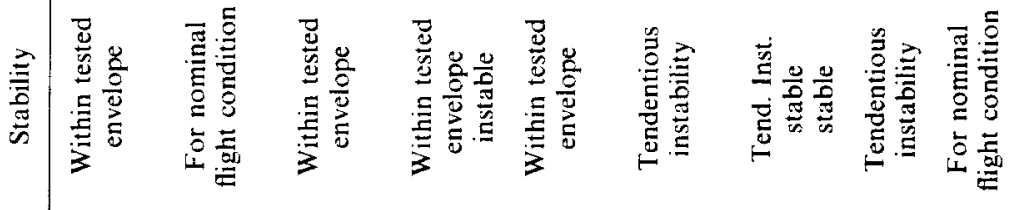

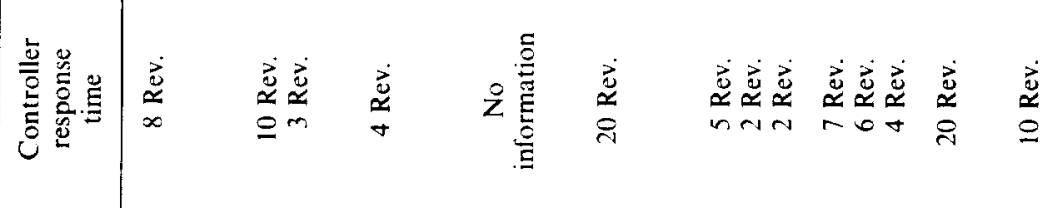

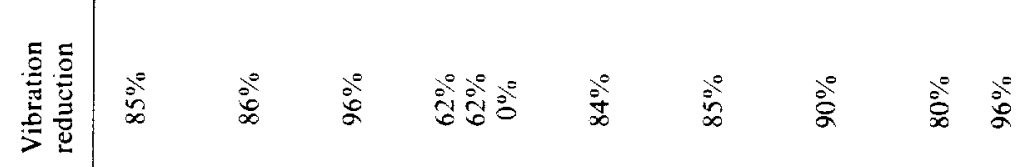

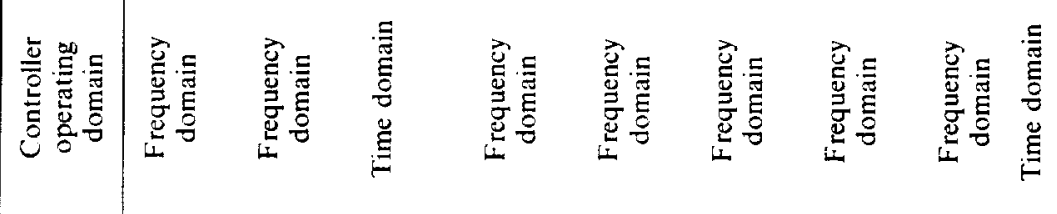

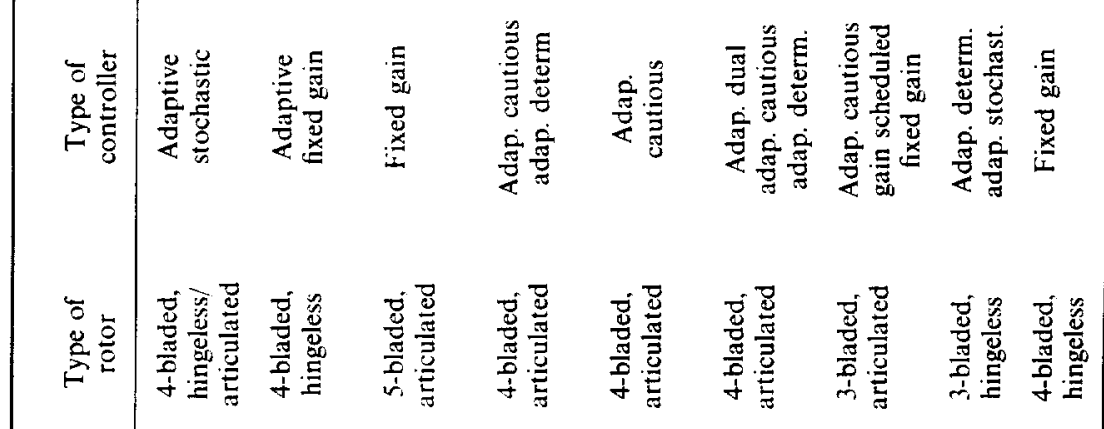

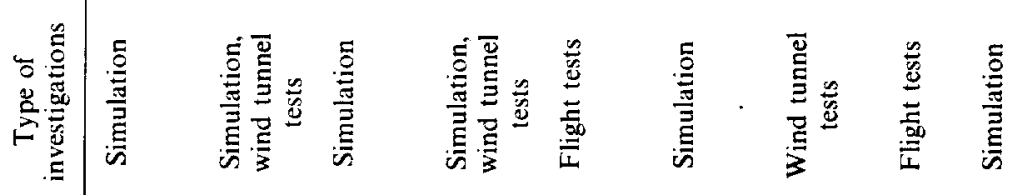

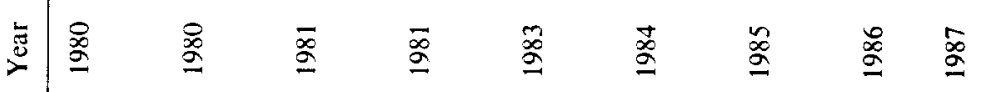

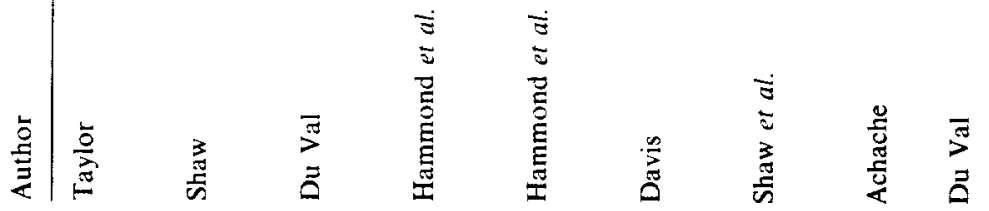




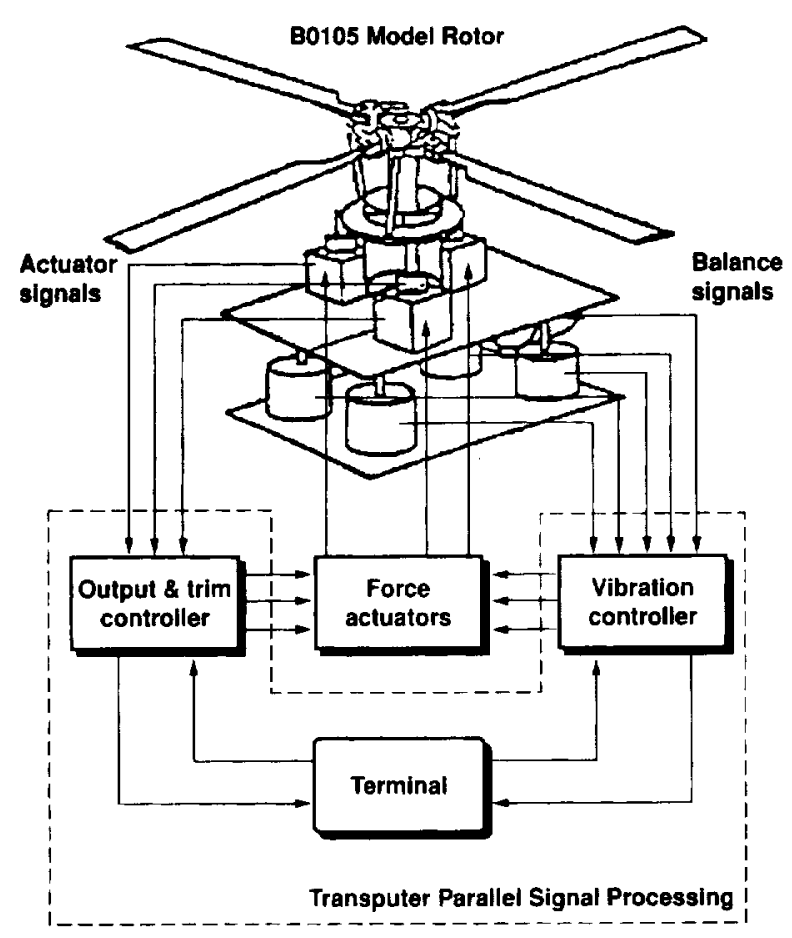

Fig. 25. Block diagram of a time domain $\mathrm{HHC}$ controller for vibration reduction. ${ }^{(4)}$

domain controller for vibration reduction is shown in Fig. 25, based on a linear time invariant representation of rotor dynamics and disturbance rejection approach for the controller design. The system response indicated that the final steady state response after a hub moment disturbance was reached within two rotor revolutions. Depending on specific applications, both frequency and time domain controllers will be required for future active rotor control systems.

\section{HHC AND IBC FOR BVI NOISE REDUCTION}

\subsection{HHC INVESTIGATIONS}

In 1985, Aerospatiale (ECF now) demonstrated in flight on a Gazelle SA 349 experimental helicopter the ability of reducing BVI noise and results were published in $1990 .^{(31)}$ Two types of measurements were conducted by using first a closed-loop HHC system for vibration reduction and second an open-loop HHC system for noise reduction. In this latter case, the purpose of the test was to determine the controls necessary to minimize noise through a systematic investigation of amplitude and phase variations of the HHC system. The synthesis of these tests is given in Polychroniadis. ${ }^{(31)}$

With a closed-loop HHC adapted for vibration reduction, Aerospatiale demonstrated a very impressive reduction of vibration (up to $80 \%$ ) on board the Gazelle. During the flight tests, noise levels were measured by microphones installed on the helicopter skids (Fig. 26) and on the ground. The most convincing results were obtained during approach at 6 slope and $28 \mathrm{~m} / \mathrm{s}$ flight velocity by using a 1 amplitude $3 P$ control (the Gazelle has a three-bladed rotor). As shown in Fig. 27, HHC removes the acoustic pressure peaks induced by BVIs. A large reduction of almost $6 \mathrm{TPNdB}$ of the maximum noise level has been measured by a microphone under the flight track $(-3.5 \mathrm{EPNdB})$. Note that the microphone on the aircraft left-hand side provides a clear view of the noise reduction obtained when HHC is 


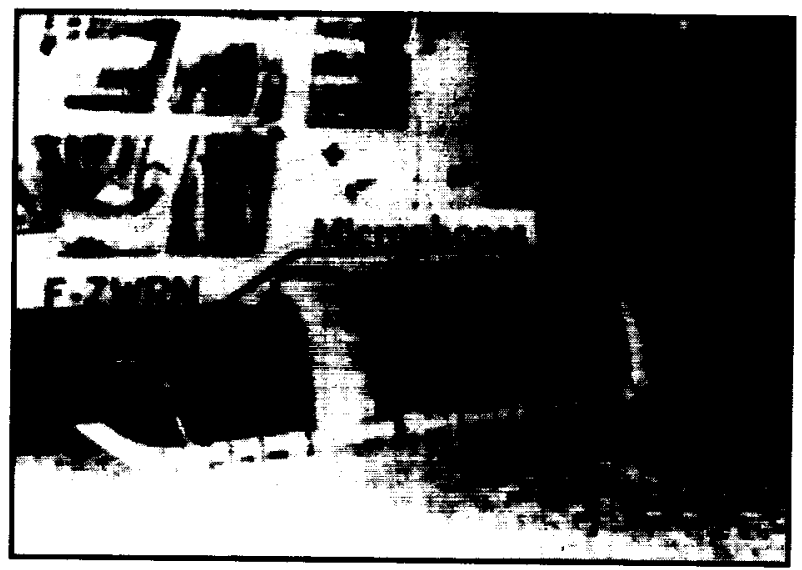

Fig. 26. Acoustic measurements on the SA349.(31)

SA 349 approach $6^{\circ}$ slope - $100 \mathrm{~km} / \mathrm{hr} /$ open loop HHC (3/Rev) Microphone on aircraft LH skid

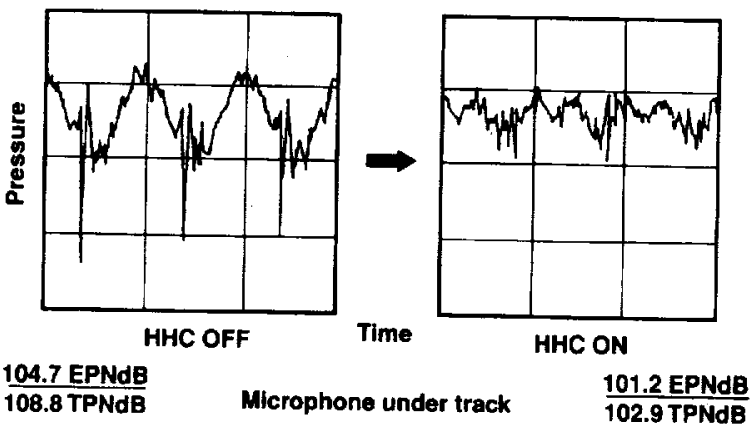

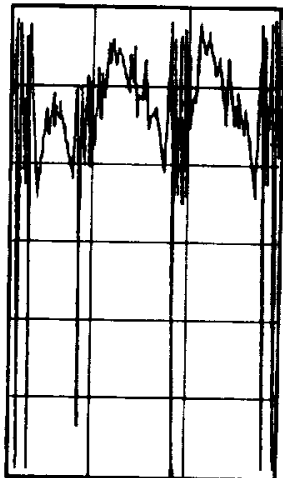

HHC OFF

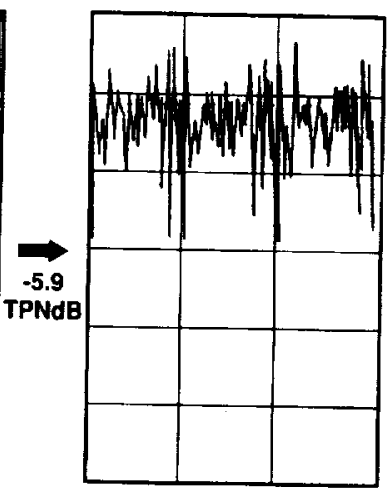

HHC ON

Fig. 27. BVI noise reduction with HHC: flight tests on SA349 t31)

working. Such a signal could probably be used in an HHC closed-loop system. This very promising 1985 result has encouraged Aerospatiale to continue research on $\mathrm{HHC}$ together with MBB, DLR, and NASA with wind tunnel tests performed at the DNW with either open-loop or closed-loop techniques.

In early 1988, initial acoustic results were obtained from a $\mathrm{HHC}$ experiment conducted in the DNW closed test section with the objective of demonstrating the effectiveness of higher harmonic blade pitch control to reduce rotor BVI impulsive noise. ${ }^{(37)}$ This test was performed as part of a HHC demonstration experiment of DLR on reduction of rotor 
induced vibrations in the fixed frame. ${ }^{(2)}$ Figure 28 shows the rotor test stand installed in the DNW $6 \times 8 \mathrm{~m}$ closed test section. The $4 \mathrm{~m}$ diameter rotor is a $40 \%$ geometrically, Mach, and dynamically scaled model of a four-bladed. hingeless BO-105 main rotor.

For the acoustic measurements, two 1/2-in microphones were installed in the closed test section: one under the advancing side and the other one under the retreating side of the rotor as shown in Fig. 28 . The microphones were placed at locations known to receive strong BVI pulse-type noise radiation under typical low-speed BVI test conditions from previous tests. Due to restrictions associated with the reverberant environment and with the neglecting of possible noise directivity changes, the results were considered more qualitative. The $3 P, 4 P$ and $5 P$ HHC modes were measured with systematic variation of the $\mathrm{HHC}$ phase angle. Significant noise reduction of the order of $5 \mathrm{dBA}$ was measured for particular HHC settings, but at the cost of increased vibration levels (Figs 29 and 30). At optimum pitch control settings for BVI noise reduction, analytical results demonstrated that at the interaction location vortex strength and blade-vortex miss distance are increased, resulting overall in reduced BVI noise generation. At optimum pitch control settings for vibration reduction, there are adverse effects on blade loading. vortex strength and blade-vortex miss distance, which explains the measured increased noise levels (of the order of $3 \mathrm{dBA}$ ).

In 1988 and 1989, a rotor test was conducted in the NASA Langley TDT to examine the use of HHC to reduce BVI noise. ${ }^{7,8)}$ A dynamically scaled, four-bladed, articulated rotor model was tested in a heavy gas ( $R-12$, nominal pressure of 0.45 atmosphere). The 110 in diameter rotor, which is dynamically scaled for R-12. has untwisted NACA 0012 section blades with a 4.24 in chord. The articulated flap and lead-lag hinges are offset 3 in from the center of rotation. As a consequence of the heavy gas, test speeds (rotor and tunnel) are reduced to 0.43 , the ratio of the speed of sound for $\mathrm{R}-12$ and air $(500 / 1130=0.43)$. The gives matched Mach number and advance ratio conditions at reduced frequency scales. Acoustic and vibration measurements were made for a large range of matched flight conditions where prescribed (open-loop) HHC pitch schedules were superimposed on the normal (baseline) collective and cyclic trim pitch. Figure 31 shows the test setup with inflow microphones mounted upstream and downstream of the rotor model. A new sound power measurement technique was developed for the reverberant field in the hard-walled tunnel. By comparing the results using $4 P$ HHC to corresponding baseline (without $\mathrm{HHC}$ ) conditions, significant mid-frequency noise reductions of 5-6 dB were found for low-speed

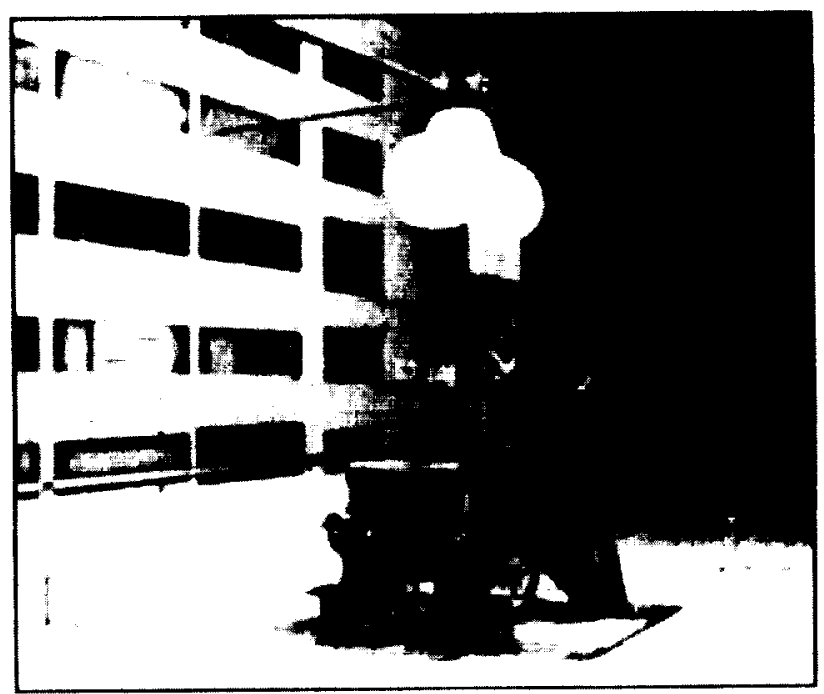

Fig. 28. DLR HHC apparatus installed in the DNW for the BVI noise reduction experiment. ${ }^{1371}$ 


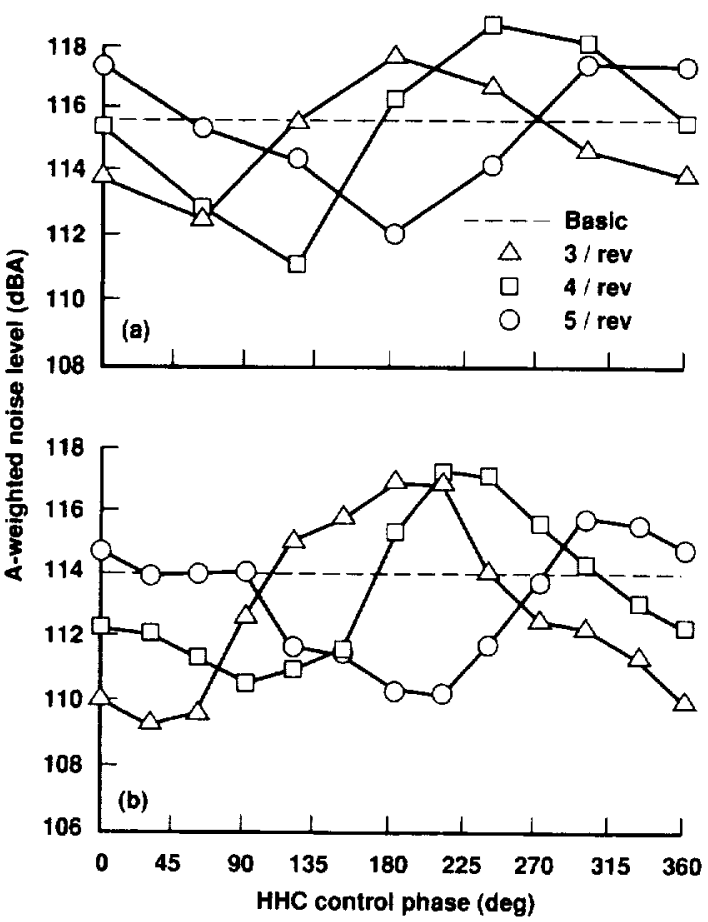

Fig. 29. HHC effect on BVI noise generation for different control modes and for two rotor test conditions at constant $M_{\mathrm{H}}=0.64$ and $C_{\mathrm{T}}=0.0044$. (a) $\mu=0.138, x_{1 \mathrm{PP}}=4.6^{\circ} ;$ (b) $\mu=0.161, x_{\mathrm{TPP}}=7.8^{\prime \prime(37)}$

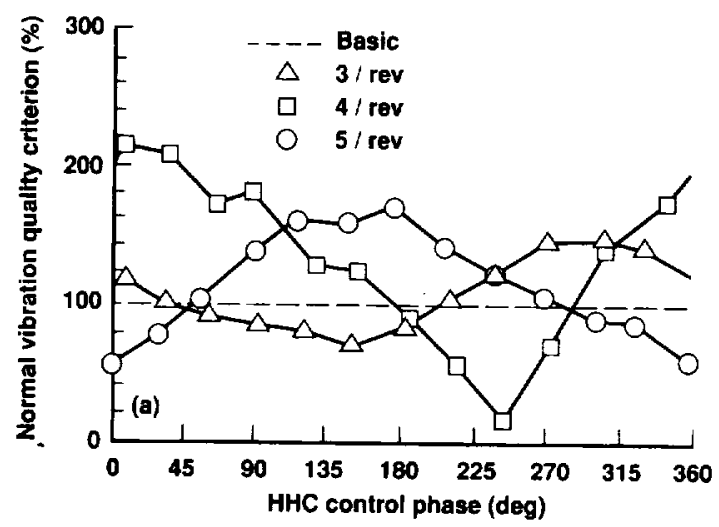

Fig. 30. Effect of $\mathrm{HHC}$ on normalized vibration quality criteria for different control modes (rotor condition as for Fig. 29(b); baseline case corresponds to $100 \%)^{(37)}$

descent conditions where BVI is most intense. For other flight conditions, noise was found to increase with the use of HHC. Low-frequency loading noise, as well as fixed and rotating frame vibration levels, showed increased levels with HHC.

The change in character of the rotor noise sources with the use of $\mathrm{HHC}$ can be observed through the instantaneous acoustic pressure time histories (Figs 32 and 33). The use of HHC diminished the BVI levels and, perhaps, the number of BVI occurrences. A large increase in the $8 P$ harmonic is clearly seen, which corresponds to the two components in Fig. 33. This study revealed the flight conditions where HHC can be used for BVI noise reduction and showed how the use of a $4 P \mathrm{HHC}$ affects the noise levels and vibratory loads. Mid-frequency noise reductions are higher (a maximum of $5.6 \mathrm{~dB}$ measured) at the lower 


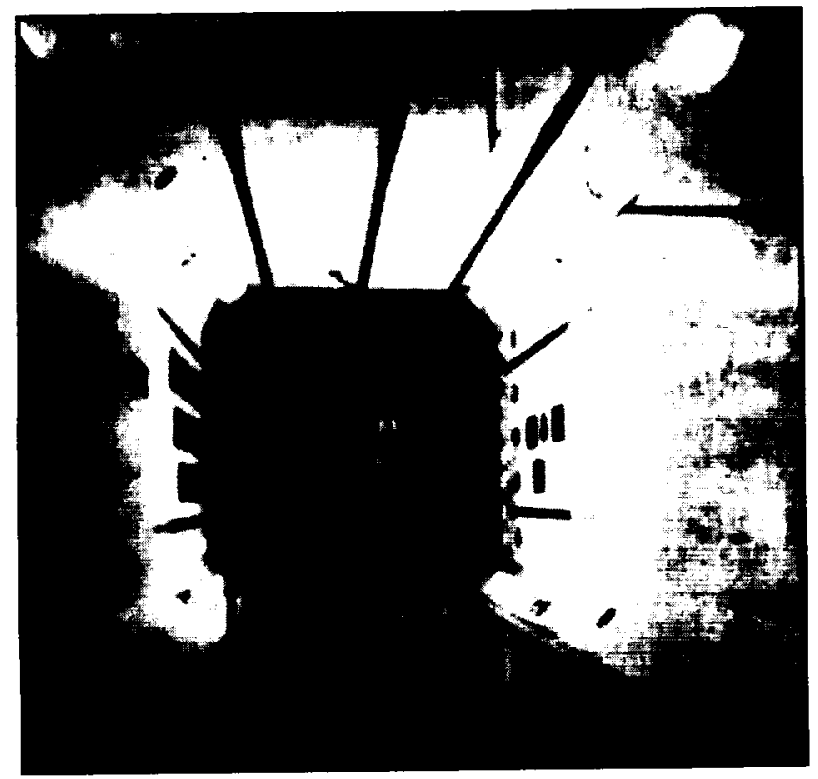

Fig. 31. Noise test set-up with microphones mounted upstream and downstream of the ARES model in the TDT ${ }^{i 81}$

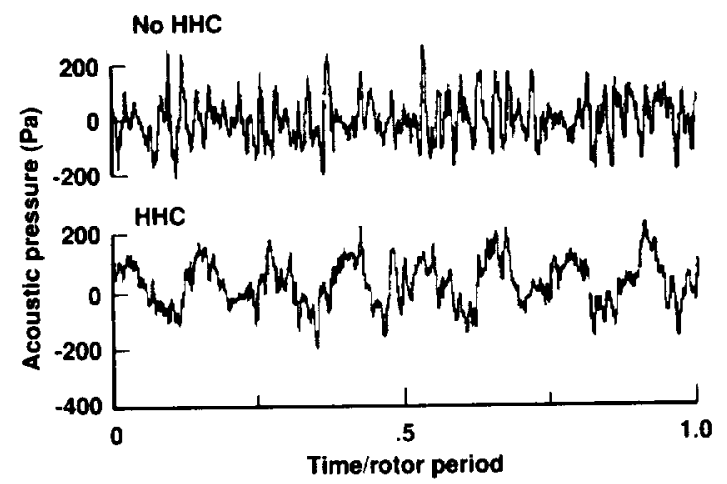

Fig. 32. Microphone signal time histories with and without HHC inputs for $\mu=0.11(\alpha=8.0)$. HHC amplitude is $\theta_{\mathrm{c}}=-1.2^{\circ}$ at $\psi_{\mathrm{c}}=60^{\circ} .{ }^{7}$

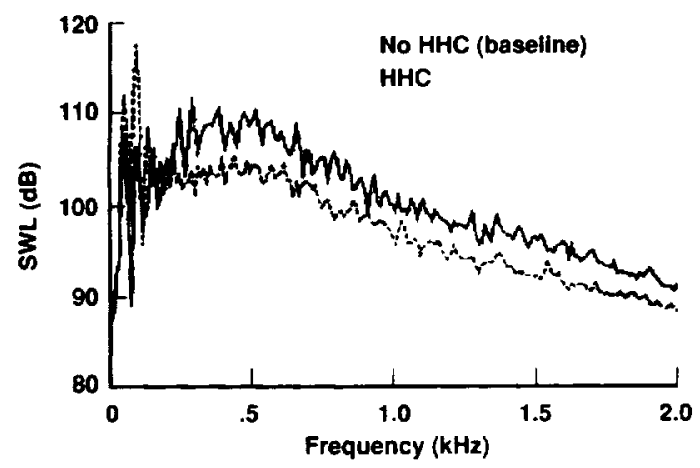

Fig. 33. Sound power spectral with and without HHC inputs for $\mu=0.11(x=8.0)$. HHC amplitude is $\theta_{\mathrm{c}}=-1.2$ at $\psi_{\mathrm{c}}=60$. Bandwidth is $11 \mathrm{~Hz} \cdot{ }^{(7)}$ 
speed descent conditions where BVI noise is most intense. No noise benefit was seen for flight conditions outside this range. For this rotor, the impulsive noise reductions correspond to reductions in blade pitch near the azimuth angle of about 60 where strong BVI is known to occur. The importance of azimuthal-dependent vortex strength changes, due to using $\mathrm{HHC}$, in the noise reductions could not be established. The use of $4 P \mathrm{HHC}$ produces increased low frequency loading noise, but its effect, as based on subjective A-weighted (dBA) measure, is not important when significant mid-frequency BVI noise reduction is attained. However, the overall significance of the increased low frequency noise should not be minimized due to other annoyance criteria and military detection concerns. The use of $\mathrm{HHC}$ was found here to increase vibratory loading.

In 1990, a three-nation cooperative HHC acoustic test was conducted in the DNW, which built upon the experience gained in France (full-scale), and in the United States and Germany (model-scale) during the early HHC acoustic testing. This scaled model rotor test was designed to further establish the noise reduction potential of $\mathrm{HHC}$ by measuring the noise directivity characteristics. The experimental approach involved the measurement of noise and vibration with and without prescribed, open-loop HHC blade pitch inputs, which comprised $3 P, 4 P$ and $5 P$ pitch schedules and some mixed modes. For the first time, an HHC rotor acoustic test was performed in an anechoic environment, which allowed uncontaminated measurements of BVI noise and the resulting influence of multicyclic pitch control on the directivity pattern. ${ }^{(9.38)}$ The DLR model rotor test rig together with a traversing inflow microphone array is shown installed in the DNW open test section in Fig. 34. The $4 \mathrm{~m}$ diameter rotor was driven by a hydraulic motor of $130 \mathrm{~kW}$ power and controlled by three electrohydraulic actuators that provided the multicyclic pitch control by proper phasing of $4 P$ input to the actuators, which moved the swashplate in the desired way. The rotor was a $40 \%$, geometrically and dynamically scaled replica of a four-bladed, hingeless BO-105 main rotor (the same as employed in Splettstoesser et al. ${ }^{(37)}$ ). The inflow microphone array was comprised of $111 / 2$-in pressure type microphones and was traversed $4 \mathrm{~m}$ forward and $4 \mathrm{~m}$ aft of the rotor hub creating a 5.4 by $8 \mathrm{~m}$ acoustic measurement plane, $2.4 \mathrm{~m}$ underneath the rotor (as illustrated in Fig. 35).

Examples of the effect of HHC variations (HHC mode, amplitude, and phase) on mid-frequency (BVI) noise level directivity are shown in Fig. 36 for a $6^{\circ}$ descent flight at $\mu=0.15$. Significant changes of the noise level and directivity were measured; the maximum

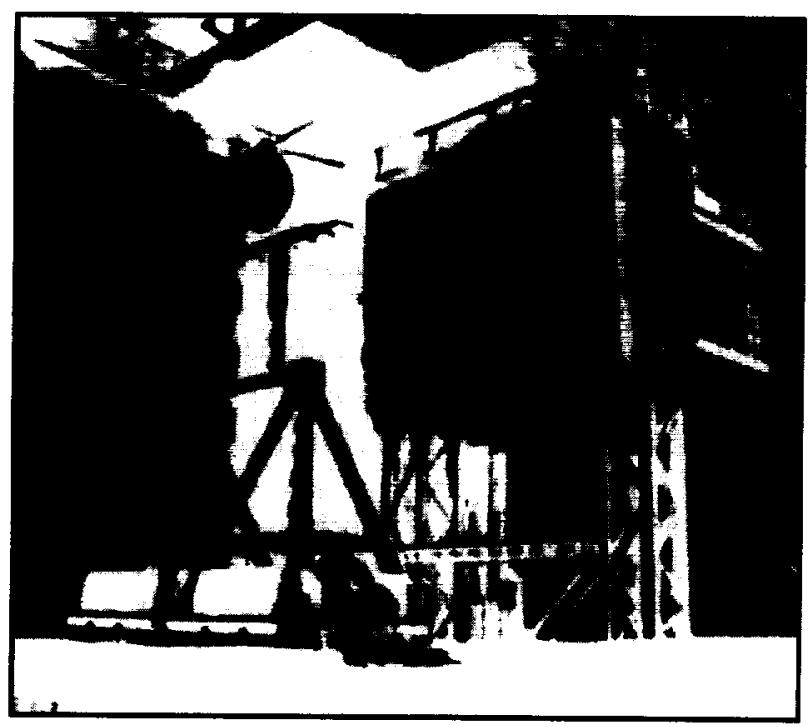

Fig. 34. Rotor model and in-flow microphone array installed in the DNW open test section. ${ }^{(38)}$ 


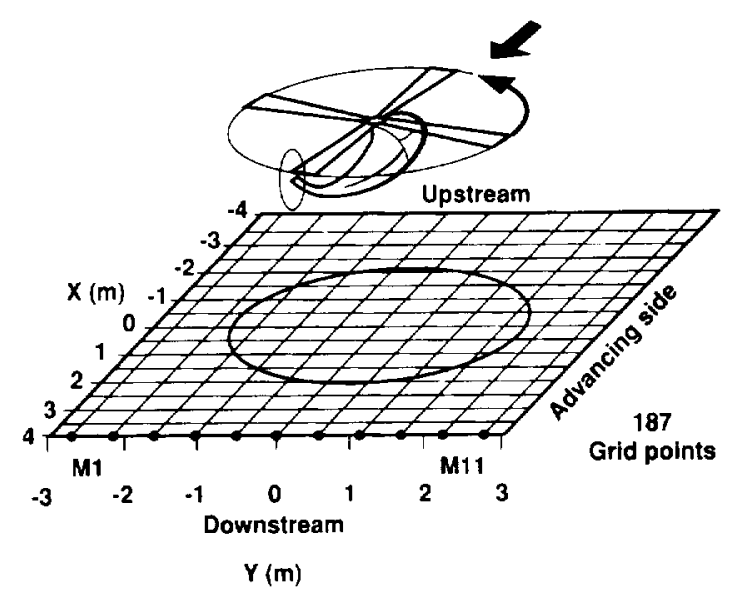

Fig. 35. Illustration of the acoustic measurement plane, 1.2R underneath the rotor hub. ${ }^{(38)}$

BVI noise level was reduced by about $6 \mathrm{~dB}$ for a $4 P$ H HC schedule with 1.2 amplitude and azimuth control angle of 54 . The maximum noise reduction was accompanied by increased low frequency noise and vibration levels, although a few HHC schedules were found in which BVI noise was reduced without an increase in vibration. A fixed pitch schedule was found to be unsuitable for all descent flight conditions generating BVI noise: to attain noise reductions over a wide range of descent angles, different $\mathrm{HHC}$ schedules had to be applied. This finding demanded application of closed-loop HHC for automatic control of BVI noise and vibrations.

Consequently, in 1991 a bilateral German French model rotor experiment in the DNW was conducted with the objective of testing a number of different closed-loop control approaches for BVI noise reduction by higher harmonic control. ${ }^{(21)}$ The test hardware was the same as in the previous open-loop HHC test performed in the DNW. ${ }^{(38)}$ A simple quadratic optimum control algorithm was insufficient for stable operation, but a more sophisticated adaptive frequency domain controller with additional vibration feedback was capable of reducing the maximum BVI level by a similar amount as for open-loop operation without increasing vibrations. A sample result is shown in Fig. 37 for a fuselage fixed microphone. Also, for non-BVI flight conditions (e.g. level flight) this adaptive frequency domain controller was stable and could be used for vibration reduction.

In order to improve the physical understanding and the mathematical modeling of the effects of the higher harmonic blade pitch control technique on impulsive BVI noise and vibration, a major cooperative research program. ${ }^{(1 .+0.49)}$ the HART program, was conducted employing a $40 \%$ geometrically and dynamically scaled BO-105 main rotor with increased blade instrumentation operated in the open-jet anechoic test section of DNW as shown in Fig. 38. Extensive efforts concerning pretest noise predictions including the effect of multicyclic pitch control were made, employing various aeroacoustic prediction codes. ${ }^{1 \prime}$ Within the experimental effort, a comprehensive set of acoustic, aerodynamic, dynamic response, performance, and rotor wake datit was acquired with a pressure and strain gauge instrumented blade and by application of nonintrusive optical measurement techniques. In agreement with previous tests, a BVI noise reduction of approximately $6 \mathrm{~dB}$ (about $50 \%$ of the maximum acoustic pressure amplitude) was obtained for $3 P \mathrm{HHC}, 0.85$ pitch amplitude, and 38 azimuth control angle (phase angle 296), termed minimum noise (MN) control (Fig. 39). Vibration reduction of about $30 \%$ was accomplished for $3 P .0 .85$ amplitude, and 119 azimuth control angle (phase angle 177 ), termed minimum vibration (MV) control. In general, at maximum noise reduction increased levels for low frequency noise and vibrations were observed, but for a small number of HHC settings both noise and vibrations were noticeably reduced. 


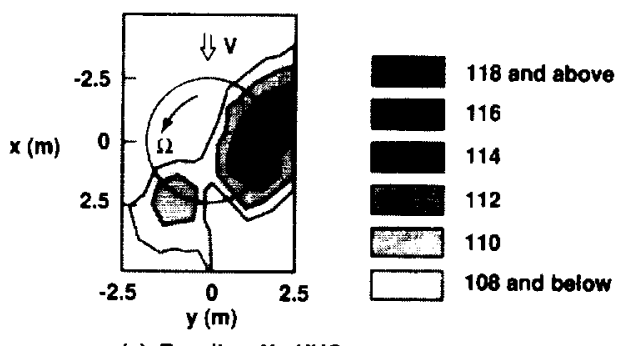

(a) Baseline, No HHC

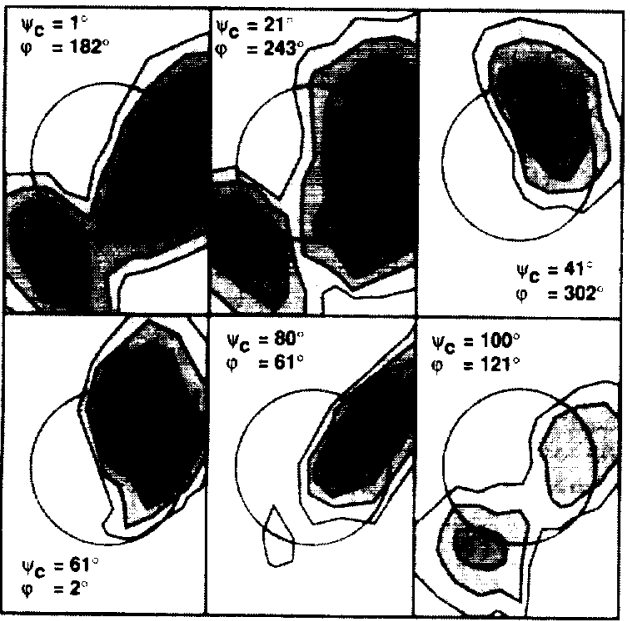

(b) 3 P HHC, $\theta_{c}=-0.8^{\circ}$

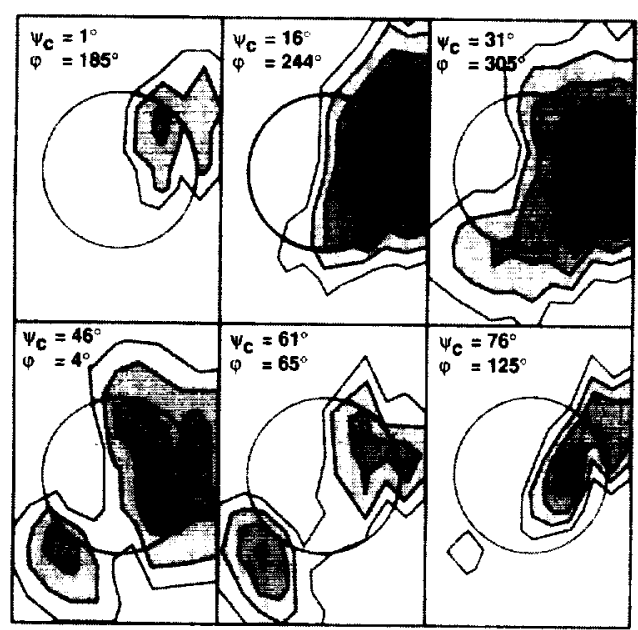

(c) 4 P HHC, $\theta_{C}=-0.8^{\circ}$

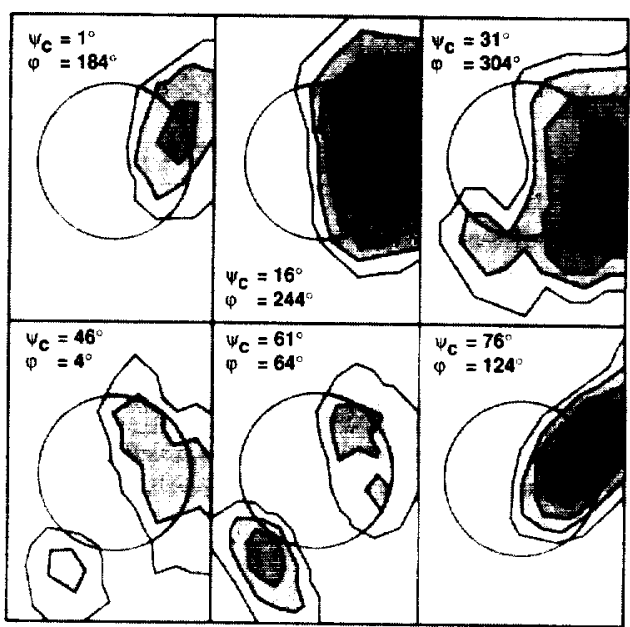

(d) 3 P HHC, $\theta_{c}=-1.2$

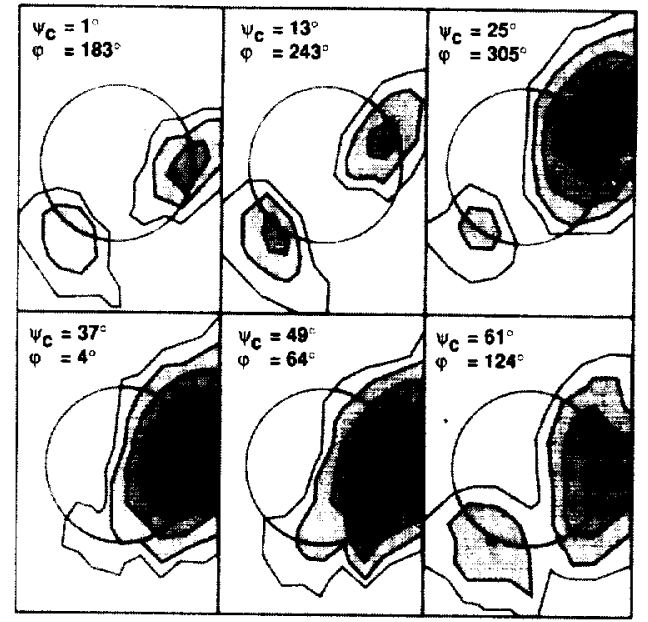

(e) 5 P HHC, $\theta_{C}=-0.8^{\circ}$

Fig. 36. Mid frequency (BVI) noise directivities for different $\mathrm{HHC}$ and phases for a $6^{\circ}$ descent flight at $\mu=0.15^{(9)}$

Absolute blade surface pressures synchronously measured with rotor performance and acoustic data were acquired for HHC rotor operation. The correlation of the unsteady blade pressure distribution and the radiated BVI noise is illustrated in Fig. 40 for a $6^{\circ}$ descent baseline case (BL) and the $3 P$ HHC cases for $\mathrm{MN}$ and $\mathrm{MV} .{ }^{(40)}$ Actual in-plane BVI locations were determined from the (near) leading edge blade pressure histories. Sectional airload or lift distributions, calculated from the blade surface pressures, were found to be extremely useful for a better understanding of the physics behind the effects of multicyclic 


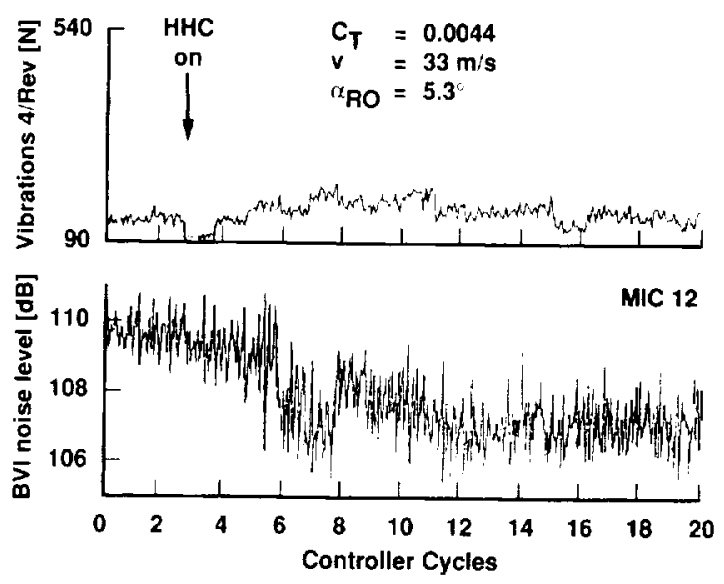

Fig. 37. Performance of sophisticated adaptive frequency domain controller with additional vibration feedback. ${ }^{211}$

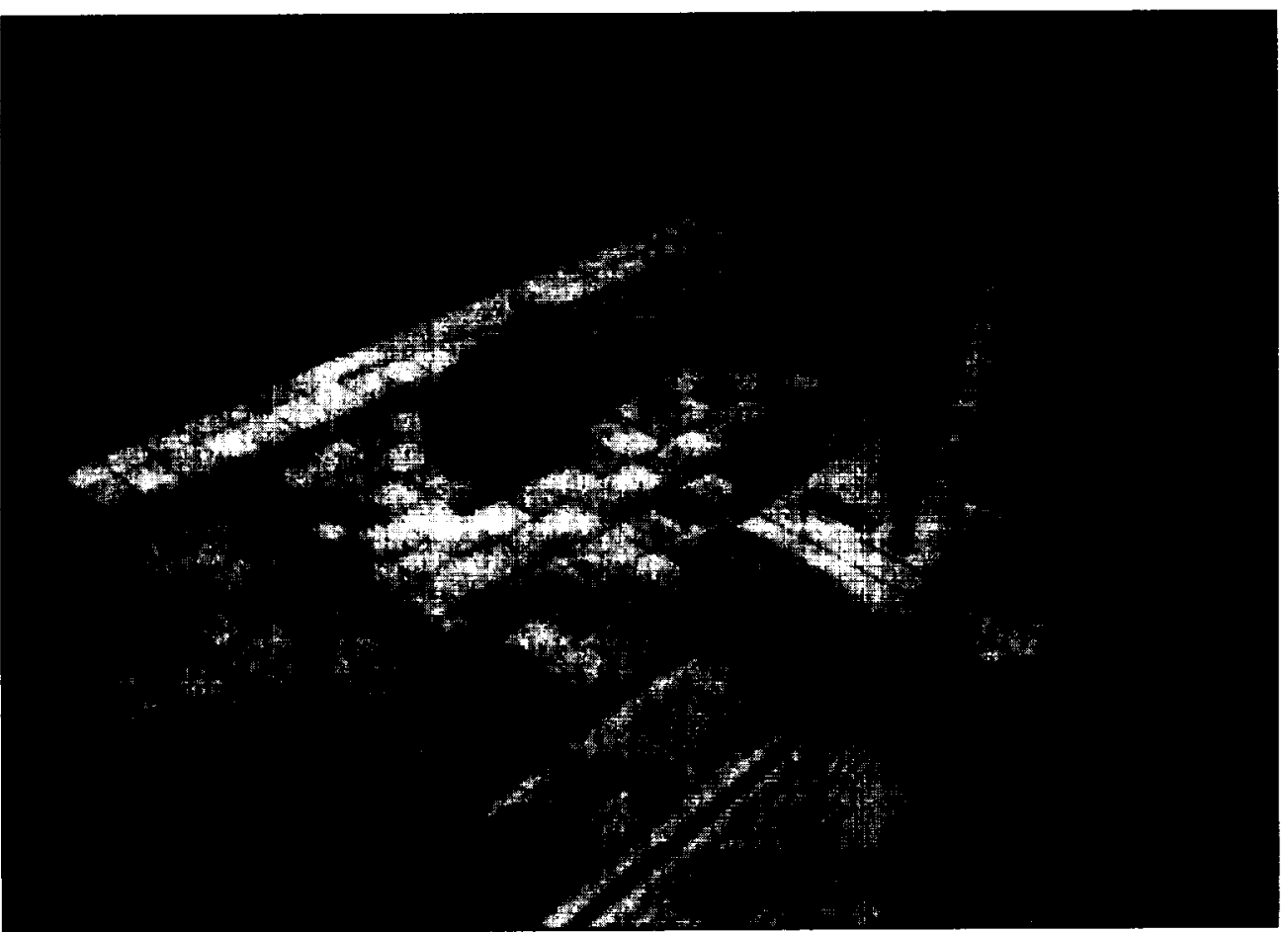

Fig. 38. Model rotor and in-flow microphone traverse in the DNW open test section. ${ }^{(40)}$

pitch variation on noise and vibration (Fig. 41). Since directly influenced by HHC, local airload represents a primary parameter for the related change of BVI noise and vibration because: (1) it determines local tip vortex strength at azimuth of vortex formation (second quadrant) and blade unloading (pitch reduction) at azimuth of blade-vortex interaction (first quadrant); (2) it controls via its induced velocity field in the blade wake (up- or downwash due to negative or positive loading) the tip vortex convection normal to the rotor plane; (3) it affects the torsional and especially the flapwise blade deflection; (4) it strongly influences the BVI geometry in general and blade-vortex miss distance in particular by the combined effects of the features of statements (2) and (3); (5) it also controls the 

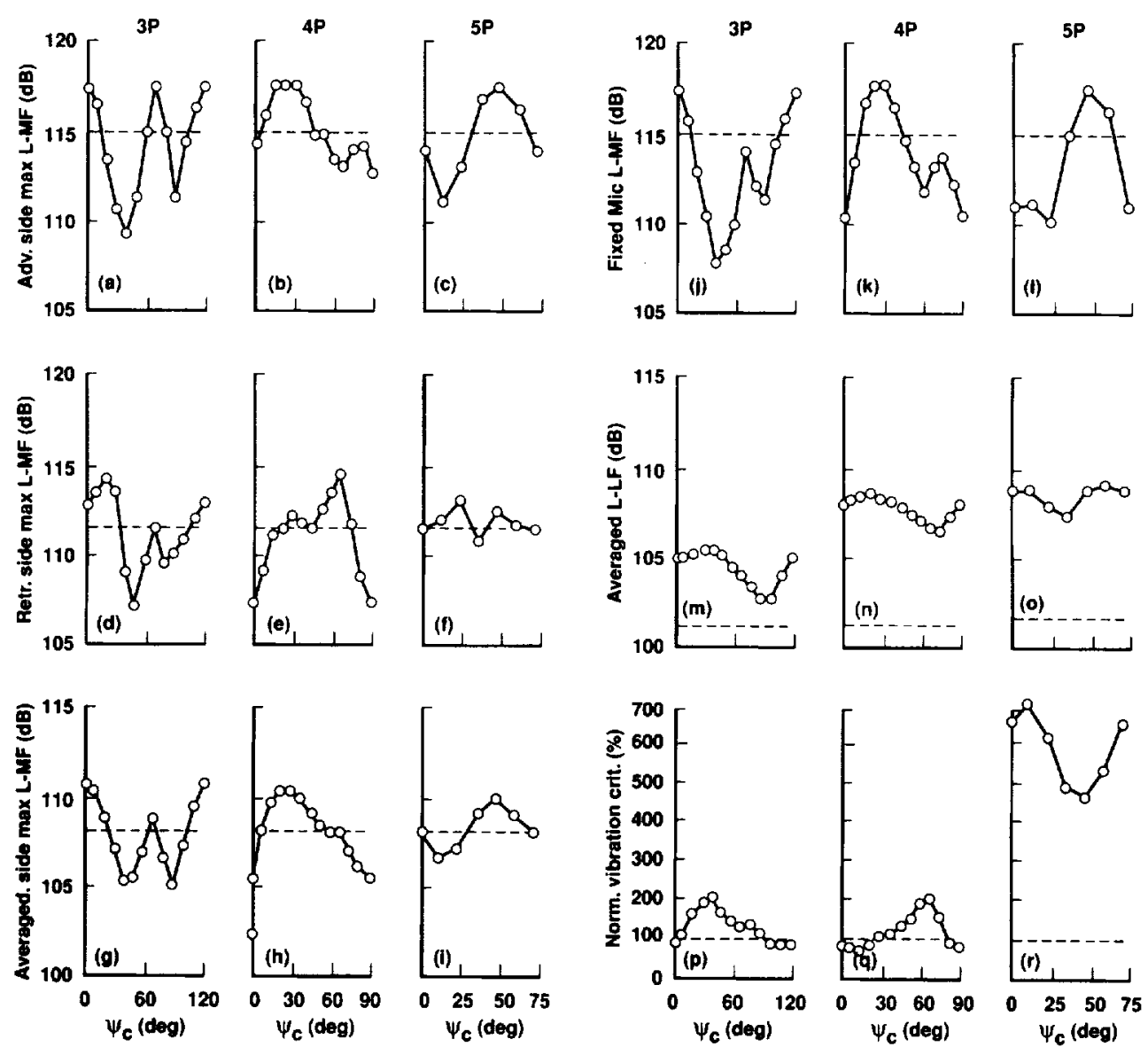

Fig. 39. Noise and vibration levels vs azimuth control angle for different HHC modes for a typical BVI condition of a 6 descent flight at $\mu=0.15$. - - for the baseline case without $\mathrm{HHC}^{(40)}$

rotor vibrations by the combined effect of the low-frequency part of the airload and the dynamic blade response and by proper phasing of the airloads the excitation of blade oscillations can be distinctly suppressed; and (6) finally, it determines the vortex formation (roll-up) and structure as seen by a double vortex structure of the MV HHC case due to negative lift on the blade tip at the azimuth of vortex emission.

Very valuable information on BVI geometry, vortex strength, and core size was obtained from the flow visualization and nonintrusive flow field measurements by the laser light sheet (LLS) and laser Doppler velocimetry (LDV) and on elastic blade deflections from applications of a projected grid method (PGM) and a target attitude in real time (TART) method. From the LLS technique, qualitative images of the tip vortex structure (e.g. double vortices for the MVcase) were attained and along with quantitative information on tip-vortex geometry segments and on blade-vortex miss distance near the azimuth of interaction. By LDV applications, the velocity vector fields of the tip vortices (one on each the advancing and retreating side) generating most intense BVI were determined. More insight into the vortex structure was gained and the double vortex system for the MV HHC case was verified. Vortex strength (circulation) of the interacting vortex was found consistent with previous results, confirming a structured distribution within the vortex, but showed only limited effect on BVI noise in this test. The retreating side values were significantly higher than on the advancing side, but they did not change much with the HHC application. Vortex core radius was determined and was observed to be $50 \%$ larger on the advancing side compared to the retreating side for the baseline descent case investigated. For MN control settings the core size was increased on the retreating side by about $70 \%$ (while core 


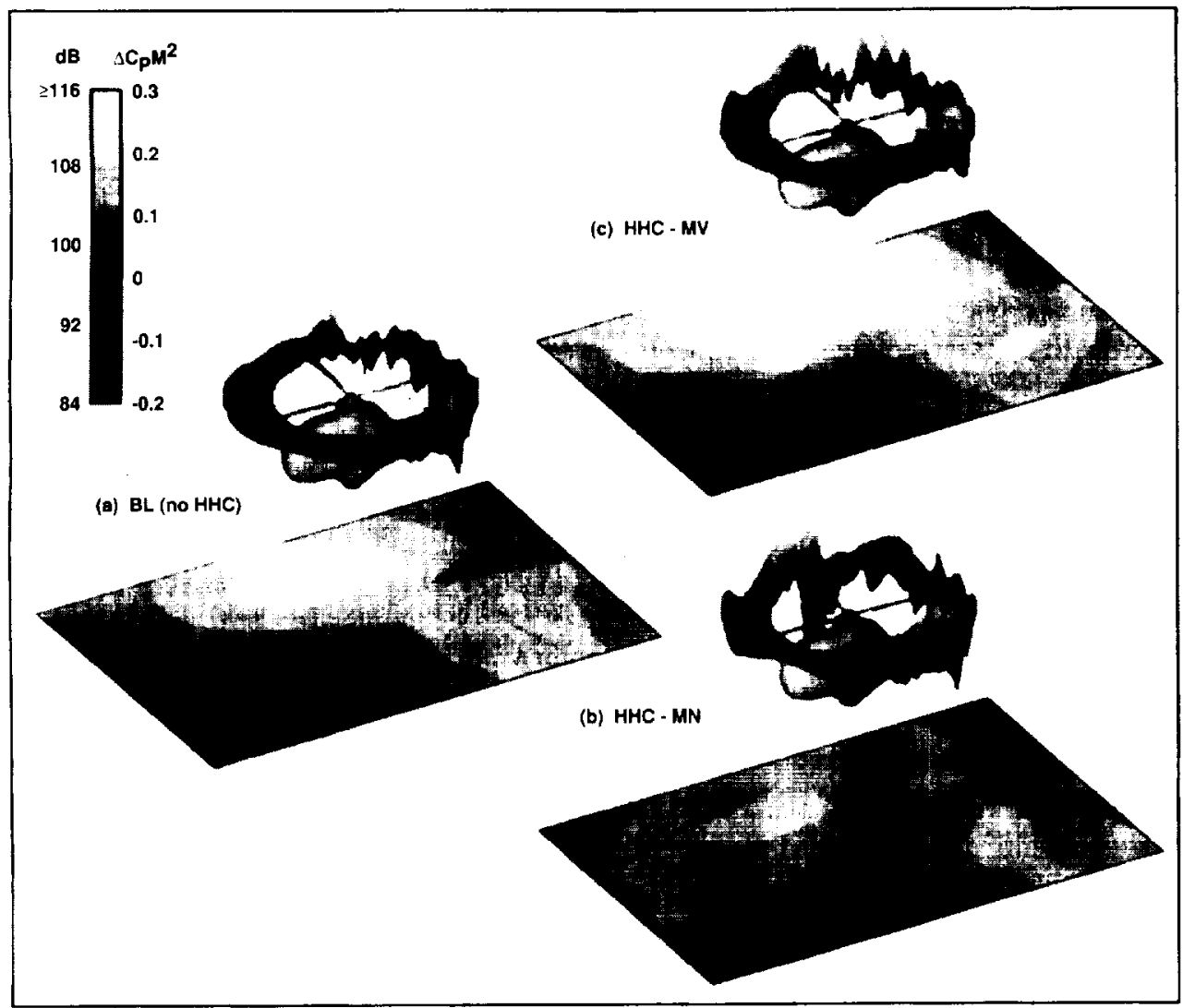

Fig. 40. High pass filtered, leading edge $\Delta C_{\mathrm{p}} M^{2}$ distributions in the rotor plane and related BVI noise contours for $\mathrm{BL} . \mathrm{MN}$ and $\mathrm{MV}$ cases for a 6 descent flight at $\mu=0.15 .^{(10)}$

(a) Baseline (no HHC)
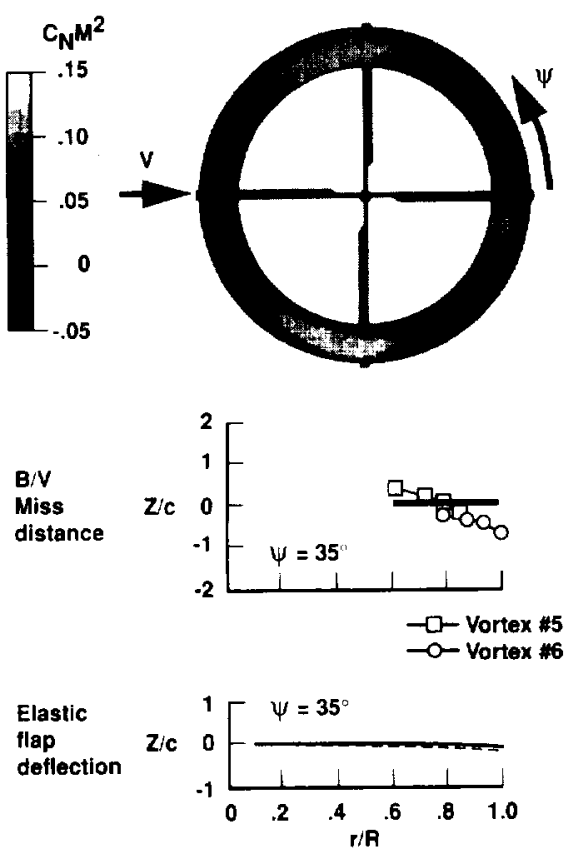

(b) HHC - MN
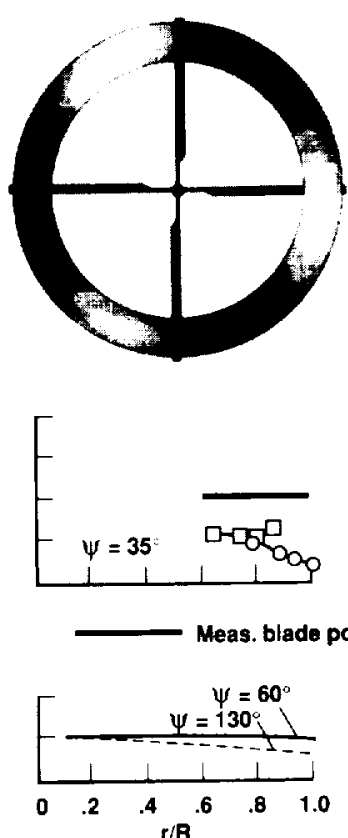

(c) HHC - MV
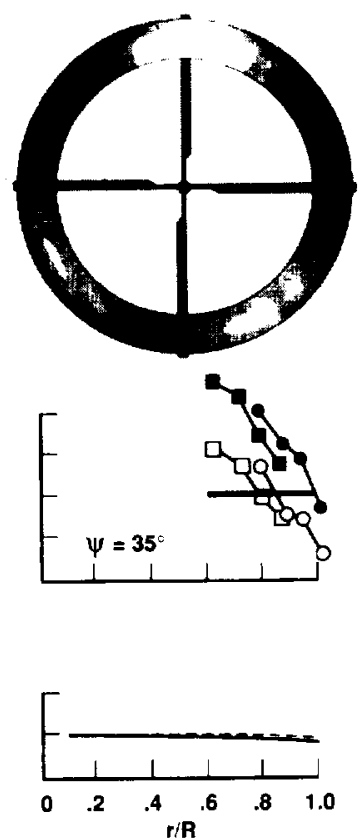

Fig 41. HHC effect on airload distribution, blade-vortex miss distance and flap deflection for a typical BVI condition of a 6 descent flight at $\mu=0.15^{(40)}$ 
size on the advancing side could not be determined). Elastic blade deflections attained along the blade span by a PGM and strain gauge measurements and at the tip by the TART method clearly showed that the blade-vortex miss distance was increased for minimum noise blade pitch control and decreased for minimum vibration.

\subsection{IBC INVESTIGATIONS}

A common feature of all HHC systems described above is that the actuators are located underneath the swashplate in the nonrotating frame. As a consequence all blades simultaneously undergo the identical pitch motion through proper phasing of the control inputs and the number of $\mathrm{HHC}$ modes is strongly restricted (e.g. due to the $4 P$ excitation of the swashplate in the case of a four-bladed rotor, only $3 P, 4 P$ and $5 P$ HHC modes are possible). This limitation is overcome by introduction of the actuators in the rotating frame, which allows IBC and a larger number of HHC modes comprising $2 P$ up to $10 P$ or more.

An IBC system, designed and manufactured by ZFL, was flight-tested jointly with Eurocopter Germany (formerly MBB) on a BO105 helicopter in 1990 and $1991 .^{(29)}$ It represented the first flying four-bladed helicopter with blades individually controlled. For safety reasons, the control authority, the flight velocity, and the loading factor were limited during the test. Further, the harmonic modes were restricted to values between $2 P$ and $5 P$ in order to reduce the number of free control parameters. Initial flight tests focusing on BVI noise reduction were conducted for $5 P$ IBC input at 0.5 amplitude and azimuth control angle of $120^{\circ}$. For a low speed, $6^{\circ}$ descent flight (a typical BVI condition) a flyover noise reduction benefit of about 4 (dBA was measured (Fig. 42). Although the initial flight tests
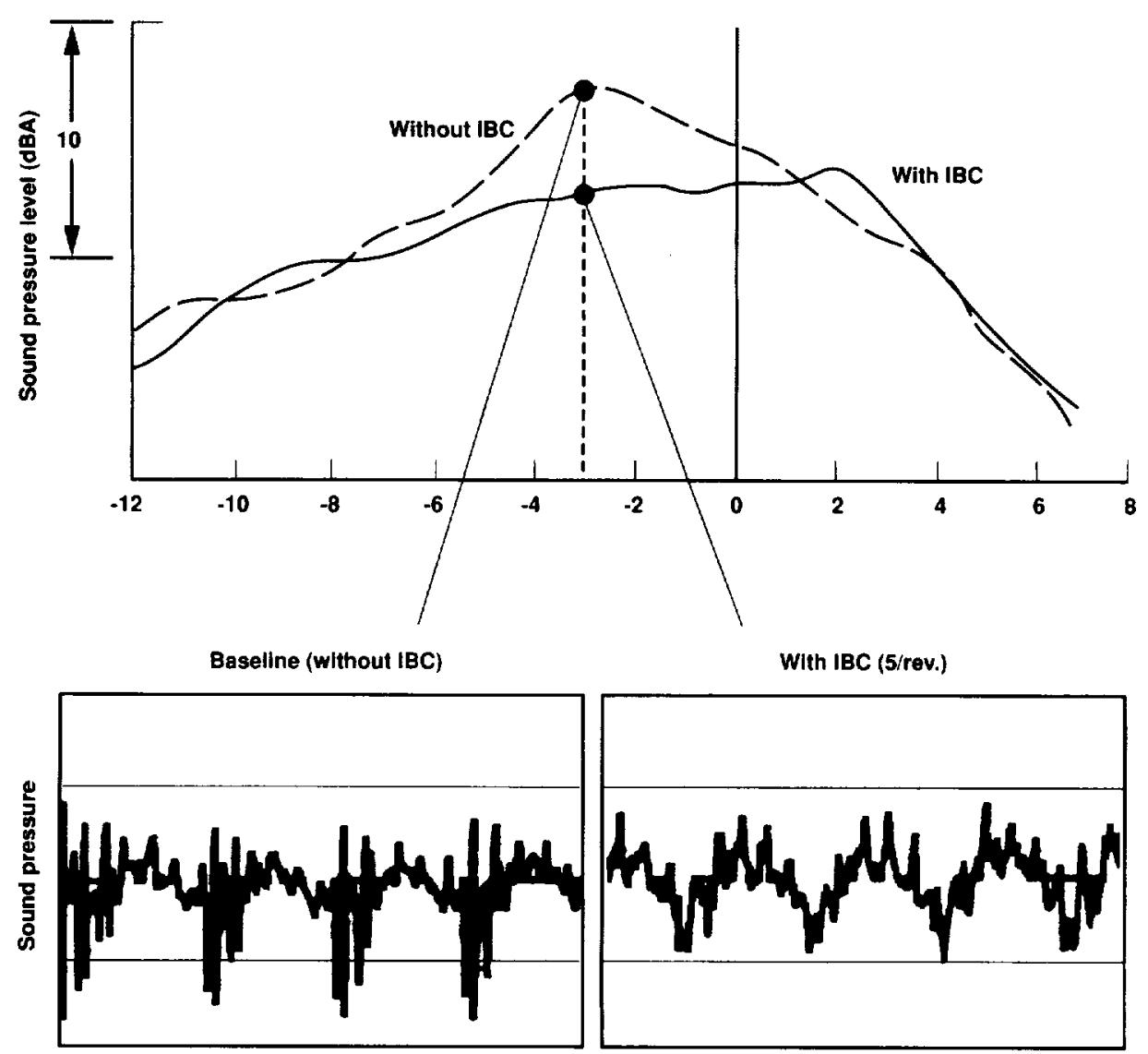

Fig. 42. Flyover noise time histories of a $\mathrm{BO}-105$ with and without $\mathrm{IBC}$ control (input mode $5 / \mathrm{rev}, 0.5^{\circ}$ amplitude, negative blade pitch at 120 azimuth, centerline microphone). ${ }^{(29)}$ 
successfully demonstrated the expected noise and vibration capability, the limited thrust and speed of the flight tests in conjunction with the low authority of the actuators prevented an exploration of the full potential of the IBC system.

Consequently, a new IBC system with higher control authority and with increased frequency response was developed and a full-scale BO-105 rotor system with an improved IBC system was tested in the 40- by 80-foot wind tunnel at NASA Ames Research Center in $1993^{(16,29,45)}$ Primary objectives of the IBC test were to evaluate the capabilities of such a system to reduce BVI noise, oscillatory loads, and rotor vibrations and to increase rotor performance. The installation of the full-scale rotor in the 40-by 80-foot closed test section is shown in Fig. 43. The IBC system was installed by replacing the four conventional rotating pitch links between the swashplate and the rotor blade with servohydraulic actuators (which werc of the same design as used for flight tests). The hydraulic power and the control signals are transmitted via slip ring devices from the nonrotating to the rotating system and then through the rotor shaft to the hub. The actuators have a stroke of $9 \mathrm{~mm}$, which results in a maximum blade pitch variation of 3 at $2 / \mathrm{rev}$ input mode. With a frequency response up to $84 \mathrm{~Hz}$, rotor harmonic frequencies up to the 12th harmonic may be excited. For this full-scale test, safety limitations restricted actuator amplitudes to 1.5 . Due to structural hardware limitations, only the 2 nd -6 th harmonic were available as single frequency inputs and to be summed as multiharmonic inputs. A conventional swashplate was employed for collective and cyclic inputs. Figure 44 shows a view of the wind tunnel test section with the microphone locations. The test section was covered by a 6 in thick acoustic absorption foam shielded by a perforated metal layer. Two microphones were mounted on the acoustic survey apparatus (ASA) below the advancing blade side of the rotor and one was installed below the retreating side. The ASA is a traversing system that allows for longitudinal travel of the microphones from $8.7 \mathrm{~m}$ before to $2.5 \mathrm{~m}$ behind the rotor hub.

For a typical BVI test condition (low speed, 6 descent) noise reductions of up to $7 \mathrm{~dB}$ were measured by different single mode IBC inputs. As shown in Fig. 45, $P P$ and $3 P$ modes were most effective and the $6 P$ input was less beneficial. Variation of the flight condition indicated (as in $\mathrm{HHC}$ applications) that a fixed pitch schedule is unsuitable for optimum noise reduction at different descent conditions, which emphasizes the need for a fast

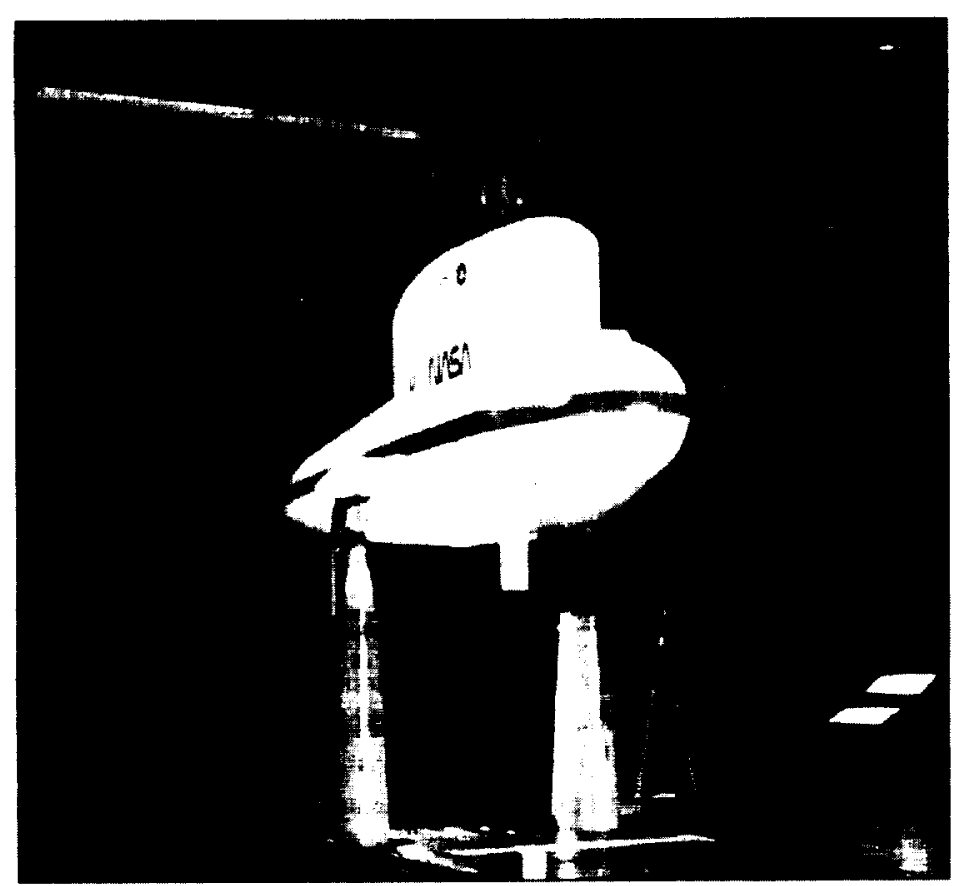

Fig. 43. BO- 105 rotor in the $40 \times 80 \mathrm{ft}$ wind tunnel. ${ }^{(29)}$ 

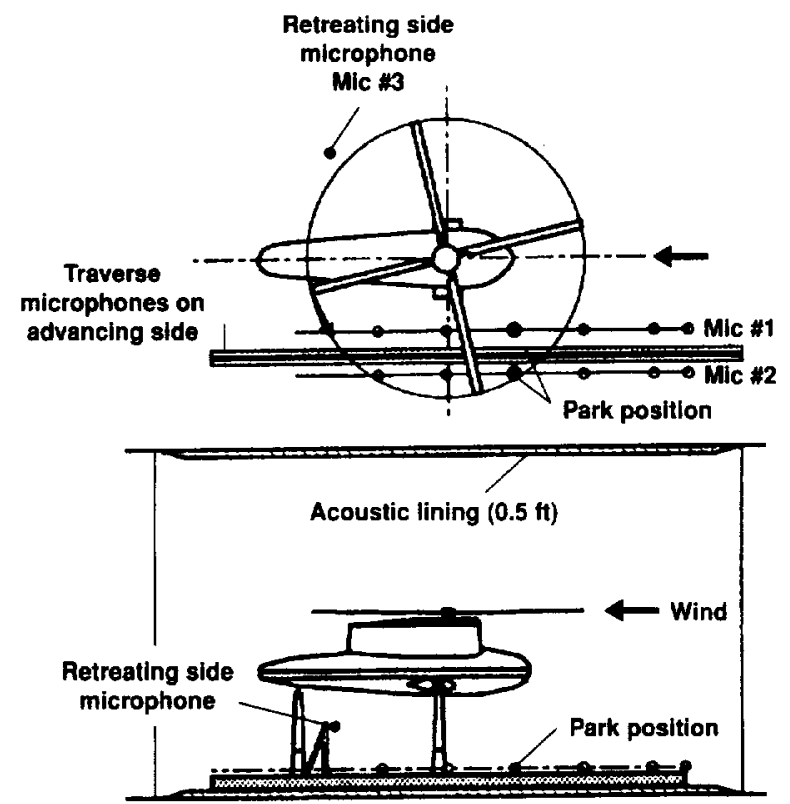

Fig. 44. Positions of the microphones and the traverse system. ${ }^{(29)}$

closed-loop controller that may be built upon feedback of an appropriate pressure sensor signal.

At an optimum IBC control setting for BVI noise reduction, some of the vibratory loads are increased. However, for an application of IBC technology on the helicopter, it is necessary to achieve BVI noise reduction without severe penalties in ride comfort. Therefore, in 1994 an IBC follow-on test was conducted in the 40 - by 80 -foot tunnel with the objective of investigating the influence of both single-harmonic and multiharmonic IBC inputs on noise, vibration, and performance. ${ }^{(17.44)}$ For all single-harmonic inputs $(3 P-6 P)$, BVI noise reductions measured at a fixed position on the order of $5 \mathrm{~dB}$ were obtained. For a $2 P$ input, a maximum reduction of about $7 \mathrm{~dB}$ was measured at $1^{\circ}$ amplitude and about $10 \mathrm{~dB}$ at $1.5^{\circ}$ amplitude for the strong BVI noise flight condition. Simultaneous reductions in acoustics $(7 \mathrm{~dB})$ and vibrations $(20 \%)$ were achieved with a $2 P$ input.

Multiharmonic IBC inputs (combined $2 P$ and $5 P$ input) achieved the best BVI noise reduction benefit, $12 \mathrm{~dB}$, at a fixed microphone location. For this combination also a maximum $90 \%$ reduction of $4 P$ vibrations was measured in comparison to the baseline (without IBC) condition.

\section{ANALYSIS OF BVI PHENOMENA AND NOISE WITH HHC}

The three parameters associated with BVI noise will be discussed, including the experimental data: blade-vortex miss distance, airloads and blade deflections. The main parameter of BVI noise is the blade-vortex miss distance as confirmed by the examination of HART experimental results. ${ }^{(1.14 .40 .49)}$ Figure 46 compares the baseline case (without $\mathrm{HHC}$ ) to the minimum noise configuration achieved from adjustment of the HHC phase setting. As shown in the figure, in the vicinity of the azimuth where blade-vortex parallel interactions occur on the advancing side (at about $\psi=50$ ), the vertical blade-vortex miss distance is increased by about $120 \mathrm{~mm}$ (i.e. is one blade chord length). These results were obtained using the LLS technique. Note that at $\psi=35^{\circ}$ the measured blade position for low noise configuration was practically the same as for the baseline case. Two mechanisms are expected to increase the blade-vortex miss distance: (1) generation of high induced 


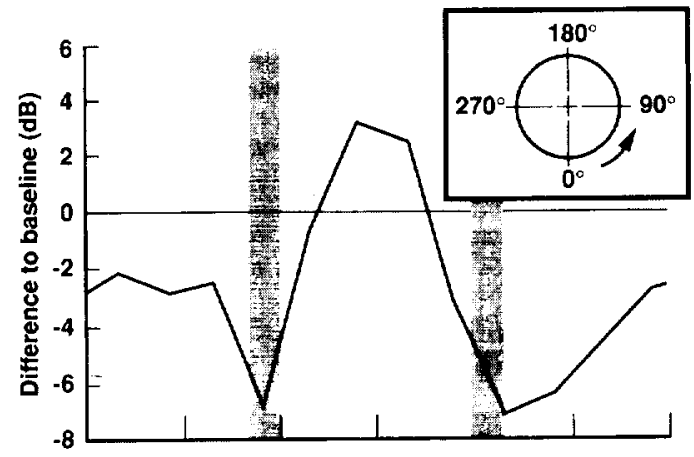

(a) $2 / \mathrm{rev}$ IBC input

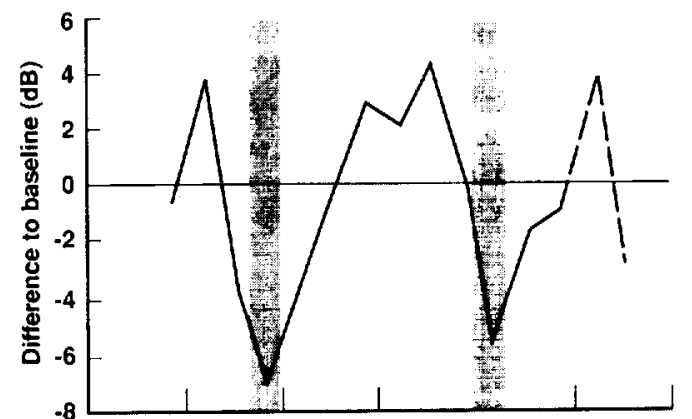

(b) 3/rev IBC input

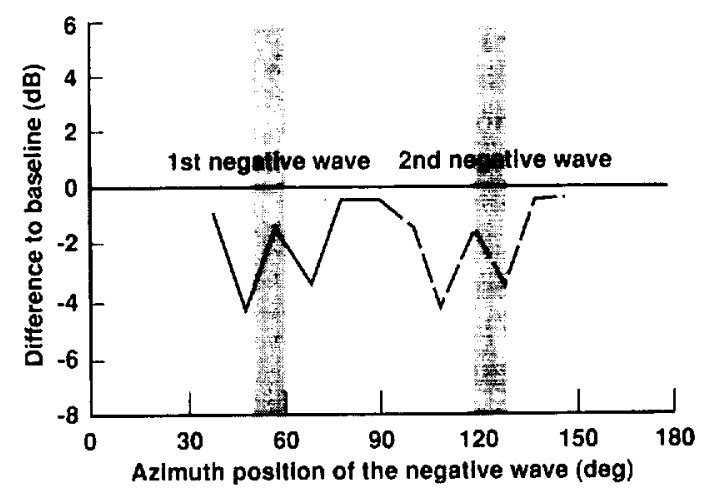

(c) 6/rev IBC input

Fig. 45. Differences of sound pressure levels to the baseline for 2-, 3- and 6/rev IBC input function vs the position of the maximum negative amplitude on the advancing blade side corrected to the tip (microphone 1, nominal fotor condition, 1 amplitude). ${ }^{(29)}$

velocities between the azimuth at which the interacting vortex is created and the azimuth of interaction with a following blade; and (2) downward deflection of the blade tip by the time the vortex is created, upward deflection at the occurrence of interaction. To assess possible contributions from these mechanisms, two kinds of experimental results from the HART tests are examined: the blade airloads derived from integration of the measured blade pressures and the blade flapping deflection.

Figure 47 compares the azimuthal distribution of blade airloads at different spanwise sections for the baseline and the minimum noise cases. As shown in the figure, when HHC is set for minimum noise, high airloads are achieved in a large azimuthal area between the generation of the noisiest vortices (about $\psi=130$ for advancing side interactions) and the interaction azimuth (about $\psi=50 \%$.

Consequently, high induced velocities are created, which push down the interacting vortex. As an example during the HART tests, with monocyclic control the airloads $C_{\mathrm{N}} M^{2}$ 


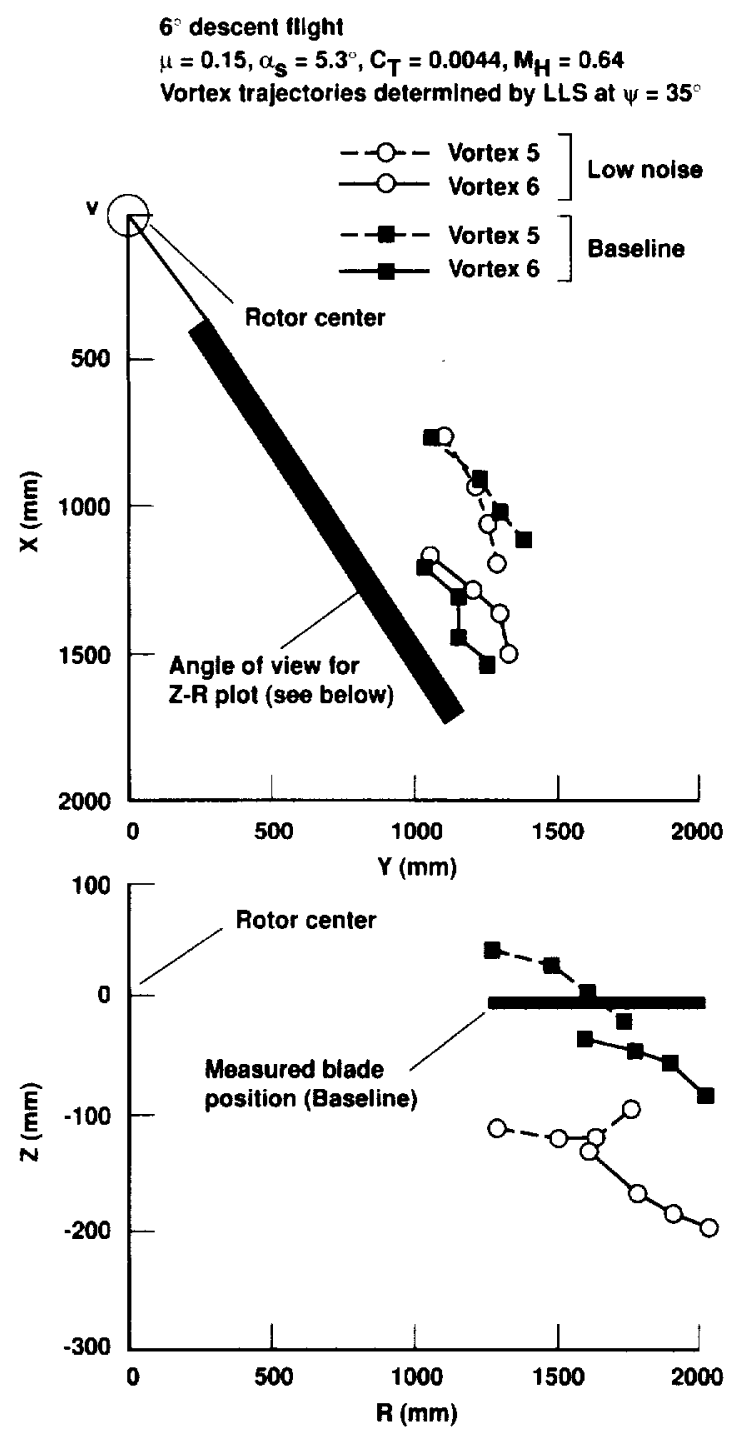

Fig. 46. Vortex trajectories determined by LLS at $\psi=35^{\circ}\left(6^{\circ}\right.$ descent flight, $\mu=0.15, x_{\mathrm{s}}=5.3, C_{\mathrm{T}}=0.0044$,

$$
\left.M_{\mathrm{H}}=0.64\right){ }^{(39)}
$$

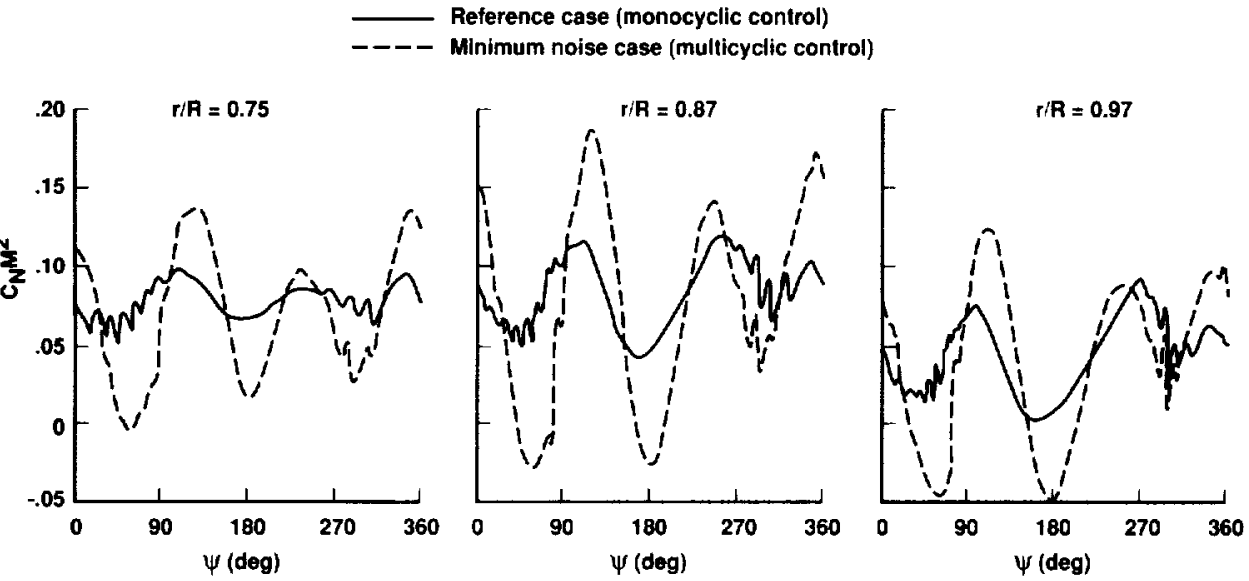

Fig. 47. Airloads on rotor blades for BVI configurations. 
at $0.87 \mathrm{R}$ varied between 0.10 and 0.11 in the $\psi=90-130$; with optimum HHC. $C_{\mathrm{N}} M^{2}$ varied between 0.10 and 0.18 . Similar trends were also found at 0.75 and $0.97 \mathrm{R}$.

Figure 48 shows, for the same HART test cases, the blade flapping deflection (resulting from both kinematics and aeroelastic deformations). Figure 48(a,b), respectively, refer to the spanwise distribution of the flapping deflection in the vicinity of the interaction azimuth and the blade tip deflection as a function of azimuth. As shown in the figures, compared to the baseline case, $\mathrm{HHC}$ at minimum noise provides: (1) a downward blade tip deflection (between 10 and $20 \mathrm{~mm}$ ) by the time the interacting vortex is generated which causes a lower location of the vortex with respect to the blade; and (2) an upward deflection $(+10 \mathrm{~mm})$ by the time the interaction occurs. Both effects tend to increase the blade-vortex miss distance at the interaction azimuth by $20-30 \mathrm{~mm}$. The blade deflection effect is, however, far too small to account for the $120 \mathrm{~mm}$ increase in blade-vortex miss distance shown by the LLS measurements. This miss distance increase is in fact, to the first-order, a consequence of the azimuthal distribution of the blade airloads achieved through adjustment of the HHC phase.

The consequences of the previous airload history and blade-vortex miss distance are: (1) a large reduction of the noise pressure peaks associated with the BVI event as illustrated in Fig. 49; and (2) a large reduction of the BVI noise levels as shown in Fig. 50, which presents the BVI noise radiation pattern below the rotor. Figure 50 shows that the maximum mid-frequency BVI noise level (related to the helicopter acoustic nuisance) can be reduced by more than $6 \mathrm{~dB}$, compared to the baseline case. It must be noted, however, that HHC increases the low-frequency noise, which can be penalizing as far as the helicopter noise detectability is concerned.

In summary, HHC has proved to be an efficient technique for BVI noise reduction in descent flight. This effect is achieved through global changes in the aerodynamics, and thus wake trajectory changes over the rotor disk which modify the geometry of the BVI. Even if stronger vortex intensities are created, the dominating effect for the noise reduction is the increase of blade-vortex miss distance. mainly due to the larger induced velocities through the rotor disk which accelerate the downward convection of the vortices.
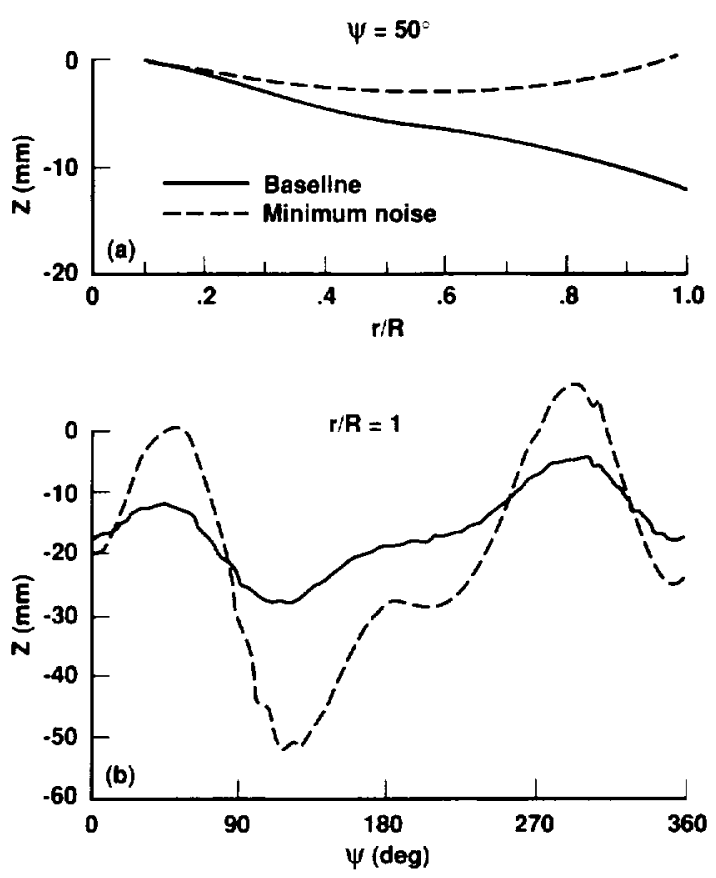

Fig. 48. Flapping deflection (without precone) ( 6 descent flight, $\mu=0.15 . x_{\mathrm{s}}=5.3, C_{\mathrm{T}}=0.0044, M_{\mathrm{H}}=0.64$ ): (a) blade deflection along the span; (b) blade tip deflection along azimuthal angle. 


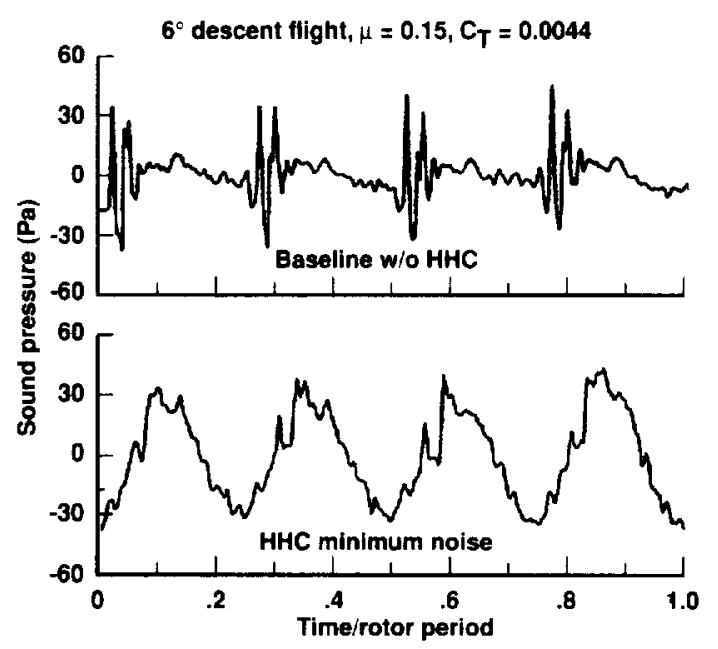

Fig. 49. Sound pressure time histories. ${ }^{(39)}$
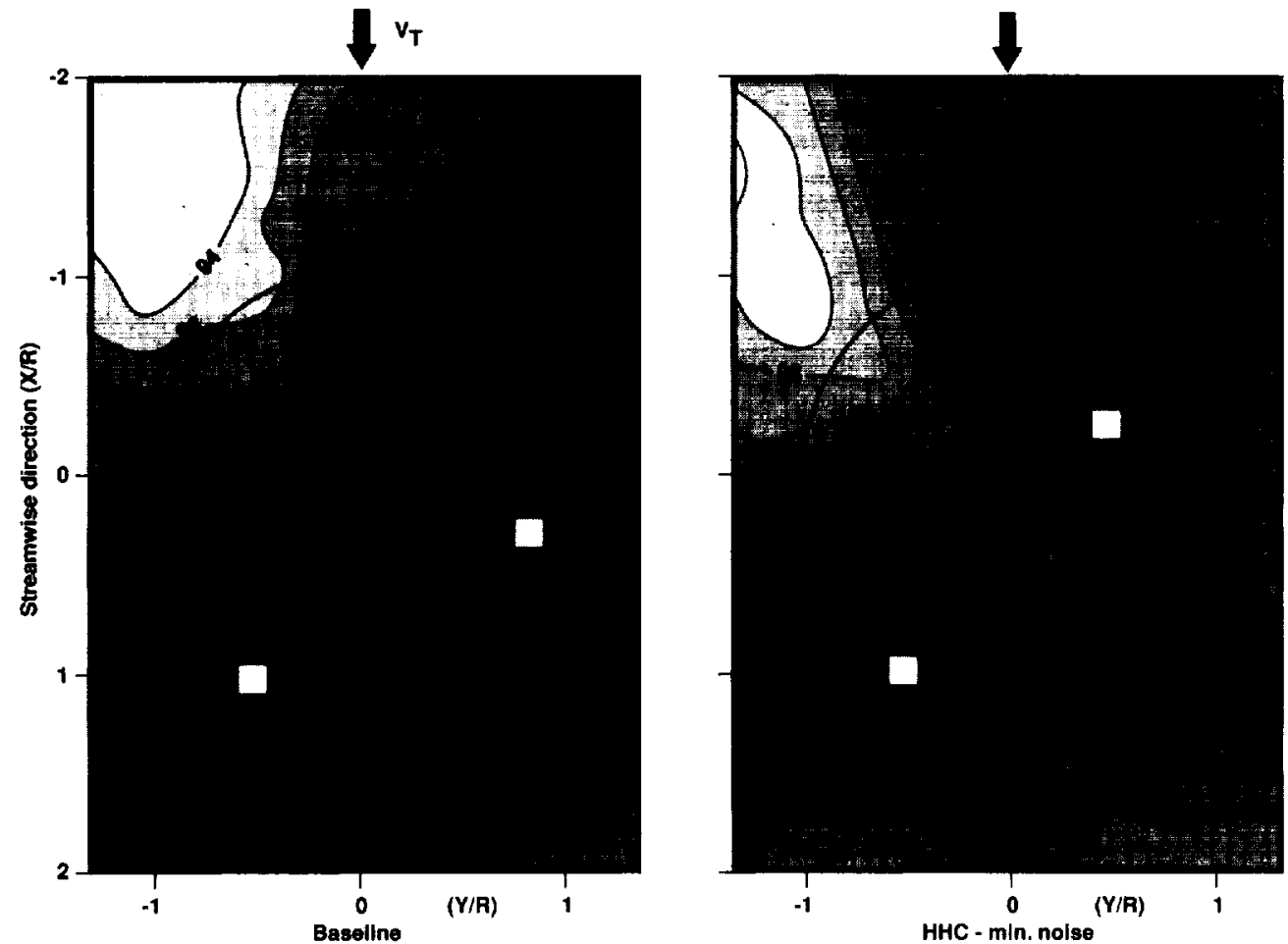

Fig. 50. BVI noise pattern below the rotor. ${ }^{(39)}$

\section{SMART ACTIVE ROTOR CONTROL CONCEPTS FOR BVI NOISE REDUCTION}

Both HHC and IBC have been investigated based on the concept of controlling the blade pitch at its root. These HHC and IBC techniques have been shown to be able to substantially reduce noise and vibration levels, but increased in some cases edgewise bending moments, torsional moments, and control loads. Furthermore, the implementation of these techniques may require additional power, weight, and complexity. Smart structures 
technology may be able to address some of these shortcomings if it is capable of exerting the large forces and moments at high frequencies. The current research to develop a smart rotor system is mainly directed at two approaches; first, embedding distributed actuators along the blade span to induce rotor twist distribution, and, second, utilizing active trailing edge flap, active vortex generator or elevon concepts.

Recently, nonharmonic deflections of the trailing edge flap (Fig. 51) were tested in the NASA Langley $14 \times 22$ foot wind tunnel to control blade tip loading distribution, which. in turn, both alters the rotor wake structure and weakens the tip vortex strength. ${ }^{(25,41)} \mathrm{A}$ flap was mechanically deflected upward by 12.5 up to 20 in limited rotor azimuth ranges. Figure 52(a) shows an acoustic time history for a baseline (non-active flap) case. which exhibits a typical BVI pressure pulse. Figure 52(b) shows the time history for the 12.5 flap motion, which exhibits no clear BVI impulse and a more broadband nature. In the BVI-dominated frequency range, the harmonic content of the signal with the flap active reduced peaks by $10 \mathrm{~dB}$ or more. However, there is an increase in low harmonic noise levels and power required for the $12.5^{\circ}$ flap case. Since helicopters have excess power available during descent, this power penalty may not in itself be a major problem to

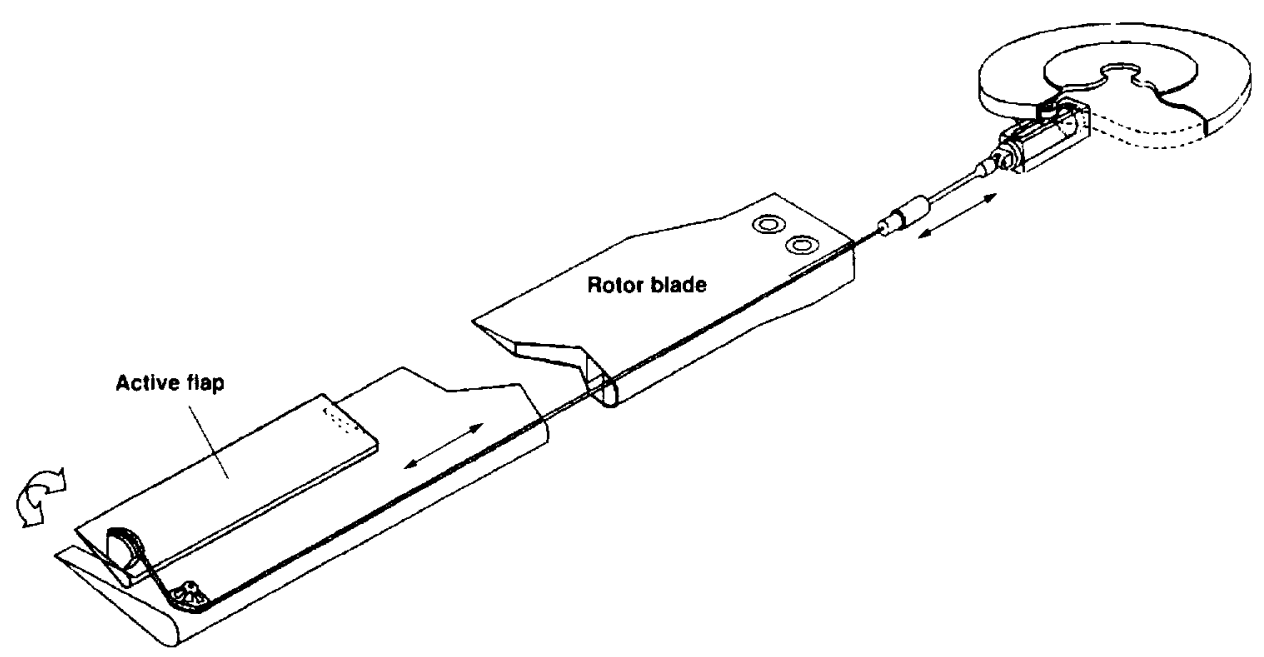

Fig. 51. Flap actuation mechanism. ${ }^{125.411}$
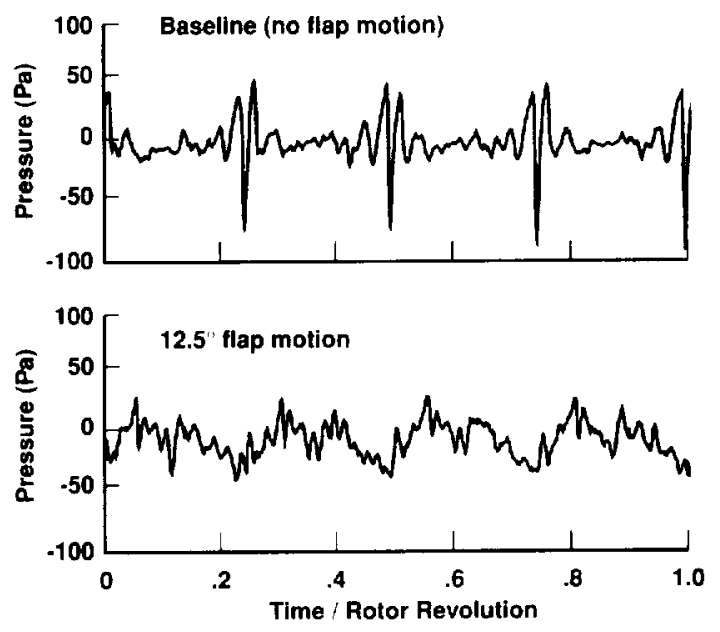

Fig. 52. Time histories at maximum BVl location in active flap rotor 1est. ${ }^{(25)}$ 
ultimate use of the concept. The broadband noise generated with the flap is also increased. In summary, a $4 \mathrm{~dB}$ decrease in the overall sound level was obtained with the active trailing flap.

Smart structures involve distributed smart actuators and sensors to apply localized strains to modify the blade shape in a controlled manner. A comprehensive review on helicopter applications was given in Chopra, ${ }^{(11)}$ Friedman and Millott, ${ }^{(12)}$ Nitzsche, ${ }^{(30)}$ Spangler and Hall, ${ }^{(36)}$ and Straub. ${ }^{(42)}$ Many types of actuators and sensors are being considered, such as piezoelectric materials, shape memory alloys, electrostrictive materials, magnetostrictive materials, electro-rheological fluids. and fiber optics. These smart materials can be integrated with rotor blades by surface bonding or embedding without causing any significant changes in the mass or structural stiffness of the blade, inducing alteration of twist/camber of airfoil that in turn will cause variation of lift distribution or modify the blade aeroelastic deformation. Two small rotor models were built and tested: ${ }^{(45)}$ active blade twist control incorporating embedded piezoceramic elements, and active trailing edge flap models with smart actuators. A 6-foot diameter four-bladed bearingless rotor model was built, where each blade was embedded with banks of specially shaped piezoelectric actuators at $\pm 45^{\circ}$ on the top and bottom surfaces as shown in Fig. 53. This rotor was tested in hover, and tip twisting of the order of 0.2 was achieved at $900 \mathrm{RPM}$ and a $4 P$ excitation. ${ }^{(10)}$ With the distributed actuators, local torsional changes and, consequently, the blade tip deflection, are induced along with flapping deformation. For the second concept, a 6-foot diameter two-bladed bearingless rotor model was built, where each blade was mounted with a trailing edge flap rotor model was built, where each blade was mounted with a trailing edge flap actuated with a piezo-bimorph. Oscillatory flap deflections up to 2 at 900 RPM have been obtained, but a major drawback was its complex hinges and

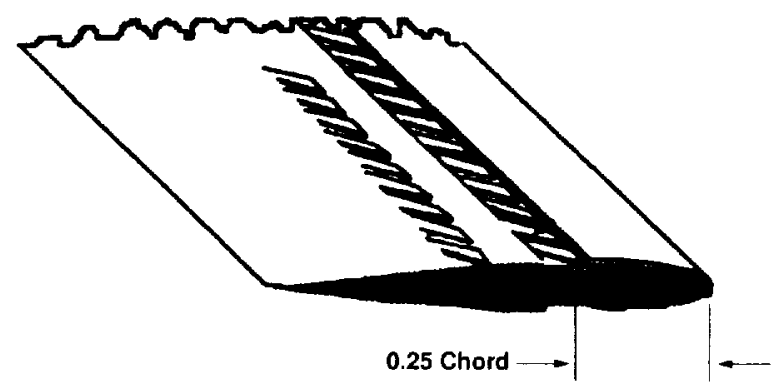

Fig. 53. Piezoceramic blade cross-section details with embedded piezo elements at $\pm 45^{(11)}$

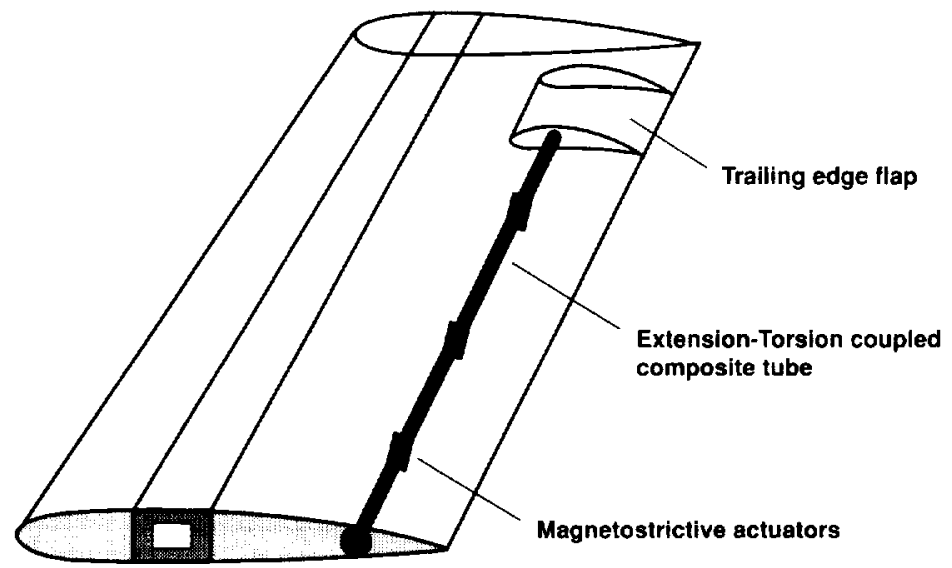

Fig. 54. Schematic of rotor blade trailing edge flap actuated by extension torsion coupled composite tube and magnetostrictive actuators. ${ }^{(3)}$ 
leverage system where considerable performance losses took place, especially at higher rotational speeds. ${ }^{(2)} \mathrm{A}$ better concept was investigated where a bending-torsion coupled composite beam with surface-bonded piezoceramic was used to actuate the flap as shown in Fig. 54. A 6-foot diameter rotor model was tested in hover, and flap deflection of 4 was obtained at 900 RPM. $^{(3)}$ However, more research has to be done before practical applications on helicopters.

\section{CONCLUSION}

Research conducted in the last decade was produced remarkable advances in understanding of the generating mechanisms of and also in controlling rotor blade-vortex interaction (BVI) noise. Several important physical parameters were identified: wake structures and trajectory, blade-vortex miss distance, rotor induced velocity distribution, and blade aeroelastic deformation.

Active rotor control techniques such as higher harmonic pitch control ( $\mathrm{HHC}$ ), individual blade control (IBC), and smart rotor control concepts have been reviewed for BVI noise reduction. The HHC technique has achieved substantial benefits in vibration reduction as well as noise reduction, but generated potential penalties such as power, weight, complexity, and limited control flexibility. However, this technology provided fundamental understanding of the active rotor control concepts to reduce BVI noise and served as an ideal basis for further developments. A comprehensive test database with this $\mathrm{HHC}$ technique is available for fundamental understanding of the noise generation and controls. The IBC technique has been developed with adequate control authority and with high frequency response. A fullscale rotor with an improved IBC system through the blade root control was tested for BVI noise and vibration reduction and achieved substantial advances in reducing simultaneously both the vibration and noise levels. Particularly, multiharmonic IBC inputs achieved the best BVI noise and vibration reduction.

Currently, the smart structures are being actively investigated to further advance the current active rotor control technology for noise and vibration reduction, such as active trailing edge flap and active blade twist control concepts with piezoelectric materials or shape memory alloys. The physical benefits generated by HHC and IBC control techniques can be produced by blade pitch or twist variations along the blade span by localized smart structure actuations in a rotating frame. potentially with much less power required. However, the capabilities of the currently available smart structures/materials are still limited for practical helicopter applications.

\section{REFERENCES}

1. Beaumier, P., Prieur. J., Rahier, G., Spiegel, P., Demargne, A., Kube, R., van der Wall, B. G., Schultz, K. J., Splettstoesser, W., Tung, C., Gallman, 3.. Yu, Y. H., Brooks, T. F., Burley, C. L. and Boyd, D. D. (1994) Effect of higher harmonic control on helicopter rotor blade vortex interaction noise: prediction and initial validation. In: 75th Fluid Dynamics Panel Symposium on Aerodynamics and Aeroacoustic's of Rotorcraft, Berlin. Germany.

2. Ben Zeev, O. and Chopra, I. (1996) Advances in the development of an intelligent helicopter rotor employing smart trailing edge flaps, In: 1995 North American Conference on Smart Structures and Materials, San Diego, CA.

3. Bernhard, A. P. F. and Chopra, 1. (1996) Development of a smart rotor with a trailing edge flap activated by a bending torsion coupled composite beam, In: 1995 North American Conference on Smart Structures and Materials, San Diego, CA.

4. Boxwell, D. A. and Schmitz, F. H. (1982) Full-scale measurements of blade vortex interaction noise, $J$. Amer. Helicopter Soc. 27 (4).

5. Brentner, K. S. and Farassat, F. (1992) Helicopter noise prediction: the current status and future direction. In: DGLR/AIAA 14th Aeroacoustics Conf., Aachen, Germany.

6. Brooks. T. F. (1993) Studies of blade vortex interaction noise reduction by rotor blade modification, In: Proc: NOISE-CON 93, Noise Control in Aeroucoustics, Williamsburg. VA

7. Brooks, T. F. and Booth, E. R. Jr (1993) The effects of higher harmonic control on blade-vortex interaction noise and vibration, I. Amer. Helicopter Soc. 35 (3).

8. Brooks, T. F., Booth. E. R., Jolly. J. R.. Yeager. W. T. and Wilbur. M. L. (1990) Reduction of blade-vortex interaction noise through higher harmonic pitch control, J. Amer. Helicopter Soc. 35 (1). 
9. Brooks, T. F., Booth, E. R., Boyd, D. D., Splettstoesser, W. R., Schultz, K. J., Kube, R., Niesl, G. H. and Streby, O. (1991) HHC Study in the DNW to Reduce BVI Noise-An Analysis. AHS/RAeS International Technical Specialists' Meeting Rotorcraft Acoustics and Fluid Dynamics, Philadelphia. PA.

10. Chen, P. C. and Chopra, I. (1995) Hover testing of a smart rotor with induced-strain actuation of blade twist, In: 36 th Structures, Structural Dynamics and Materials Conference, Adaptice Structures Fortum, New Orleans. LA.

11. Chopra, L. (1996) Review of current status of smart structures and integrated systems, SPIE 2717, 20-62.

12. Friedman, P. P. and Millot1, T. A. (1994) Vibration reduction in rotorcraft using active control -a comparison of various approaches, In: 1994 American Helicopter Society Aeromechanics Specialists' Conference, paper 7.6, San Francisco, CA

13. George, A. R. (1978) Helicopter noise-state-of-the-art, J. Aircr. 15(11), 707-715.

14. Gmelin, B. L., Heller, H. H., Mercker, E., Philippe, J. J., Preisser, J. S. and Yu, Y. H. (1995) The HART programme, a quadrilateral cooperative research effort, In: 51st American Helicopter Society Annual Forum. pp. 695-710, Ft Worth, TX.

15. Hardin, J. C. and Lamkin, S. L. (1986) Concepts for reduction of blade vortex interaction noise, J. Aircr. 24(5), pp. $120-125$

16. Jacklin, S. A., Nguyen, K., Blaas, A. and Richter, P. (1994) Full scale wind tunnel test of a helicopter individual blade control (IBC) system. In: 50th Annual Forum of the American Helicopter Society.

17. Jacklin, S. A., Blaas, A., Teves, D. and Kube, R. (1995) Reduction of Helicopter BVI Noise, Vibration, and Power Consumption Through Individual Blade Control. 5/st Annual Forum of the American Helicopter Society:

18. Kretz, M. and Larche, M. (1980) Future of helicopter rotor control, Vertica 4 (1).

19. Kube, R. (1990) Evaluation of a constant feedback gain for closed loop higher harmonic control, In: 16 th European Rotorcraft Forum, Glasgow, U.K.

20. Kube, R. and Schultz, K. J. (1996) Vibration and BVI noise reduction by active rotor control: HHC compared to IBC, In: 22nd European Rotorcraft Forum, Brighton, U.K.

21. Kube, R., Achache, M., Niesl, G. and Splettstoesser, W. R. (1992) A closed loop controller for BVI impulsive noise reduction by higher harmonic control, In: 48th Annual Fortum of the American Helicopter Society. Washington, D.C.

22. Lehmann, G. and Kube, R. (1988) Automatic vibration reduction at a four-bladed hingeless model rotor $\cdots$ a wind tunnel demonstration, In: 14th European Rotorcraft Forum, Paper no. 60.

23. Lorber, P. F. (1990) Aerodynamic results of a pressure-instrumented model rotor test at the DNW, In: $46 t h$ Annual Forum of the American Helicopter Society, Washington, D.C

24. Lowson, M. V. (1991, 1992) Progress towards quieter civil helicopters, In: 17th European Rotorcraft Forum. Berlin, Germany, Paper no. 91-59 and Aeronat. J. 209-223.

25. Marcolini, M. A., Booth, E. R., Tadghighi, H., Hassan, A. A., Smith, C. D. and Becker. L. E. (1995) Control of BVI noise using an active trailing edge flap. In: 1995 AHS Vertical Lift Aircraft Design Conference, San Francisco, CA.

26. Marze, H. J. and Philippe, J. J. (1994) A quiet helicopter--a research programme today, a reality tomorrow, In: 20th European Rotorcraft Forum. Amsterdam, Netherlands, Paper no. 9.

27. McCloud, J. L. (1980) The promise of multicyclic control, Vertica 4 (1).

28. Mercker, E. (1994) Compilation of test data for the HART-test, DNW Technical Report no. 94.03. Amsterdam. Netherlands.

29. Niesl, G., Swanson, S. M.. Jacklin, S. A., Blaas, A. and Kube. R. (1994) Effect of individual blade control on noise radiation, In: AGARD Aeroacoustics Conference, Berlin, Germany.

30. Nitzsche, F. (1995) Tailoring the dynamic response of helicopter rotors using smart materials, In: AIAA/ASME Adaptive Structure's Forum, AIAA 95-1083, New Orleans, LA

31. Polychroniadis, M. (1990) Generalized higher harmonic control - ten years of aerospatiale experience, $l 6 t h$ European Rotorcraft Forum, Glasgow, U.K.. Paper no. III 7.2.

32. Polychroniadis, M. and Achache. M. (1986) Higher harmonic control: flight tests of an experimental system on SA349 research gazelle, In: 42nd Annual Forum of the American Helicopter Society' Washington, D.C.

33. Richter, P., Eisbrecher, H. D. and Kloeppel, V. (1990) Design and first tests of individual blade control actuators, In: 16th European Rotorcraft Forum, Glasgow, U.K.

34. Schmitz, F. H. and Yu, Y. H. (1986) Helicopter impulsive noise: theoretical and experimental status, J. Sound Vibr. 109(3), 361-422.

35. Shockey, G. A., Williamson, T. W. and Cox, C. R. (1976) Helicopter aerodynamics and structural loads survey In: 32nd Annual Forum of the American Helicopter Society, Washington, D.C.

36. Spangler, R. L. Jr and Hall, S. R. (1990) Piezoelectric actuators for helicopter rotor control, In: 3/st AIAA Structures, Structural Dynamics and Material Conference.

37. Splettstoesser, W. R., Lehmann, G. and van der Wall, B. $(1990,1989)$ Higher harmonic control of a helicopter rotor to reduce blade-vortex interaction noise. Zeitschrift Fuer Flugwwissenschaften 14, 109-116 and In: 15th European Rotorcraft Forum.

38. Splettstoesser, W. R., Schultz, K. J., Kube, R., Brooks, T. F., Booth. E. R., Niesl, G. and Streby, O (1994) A higher harmonic control test in the DN to reduce impulsive BVI noise, J. Amer. Helicopter Soc. 39 (4).

39. Splettstoesser, W. R., Kube, R.., Seelhorst, U., Wagner, W.. Boutier, A., Micheli, F.. Mercker, E. and Pengel, K (1995) Higher harmonic control acroacoustic rotor test (HART)-test documentation and representative results, DLR Report no. DLR-IB-129-95/28, Braunschweig, Germany.

40. Splettstoesser, E. R., Kube, R., Wagner, W., Seelhorst, U., Boutier, A., Micheli, F., Mercker, E. and Pengel, K (1997) Key results from a higher harmonic control aeroacoustic rotor test (HART) in the German-Dutch wind tunnel, J. Amer. Helicopter Soc. 42 (1).

41. Straub, F. K. (1993) A feasibility study of using smart materials for rotor control, In: 49 th Annual Forum of the American Helicopter Society, St Louis, MO. 
42. Straub. F. (1995) Application of smart materials to rotor control, In: Second Workshop on Smart Structures and Materials, University of Maryland, College Park. MD.

43. Straub, F. K. and Byrns. E. V. (1986) Application of higher harmonic blade feathering on the $\mathrm{OH}$-6A helicopter for vibration reduction, NASA CR-4031.

44. Swanson, S. M., Jacklin, S. A., Blaas, A., Niesl, G. and Kube, R. (1995) Acoustic results from a full-scale wind tunnel test evaluating individual blade control, In: $5 /$ st Annual Forum of the American Hclicopter Society. Fort Worth, TX.

45. Swanson. S. M.. Jacklin. S. A.. Blaas. A.. Kube. R. and Niesl, G. (1994) Individual blade control effects on blade vortex interaction noise. In: 50th Ammul Forum of the American Helicopter Society.

46. Tangler, J. L. (1977) Schlieren and noise studies of rotors in forward flight, In: 33rd AHS National Forum, Washington, D.C., Paper no. 33-05.

47. Teves, D.. Niesl, G., Blaas, A. and Jacklin. S. (1995) The role of active control in future rotorcraft, In: 2lst European Rororcraft Forum. St Petersburg. Russia.

48. Walsh, D. M. (1986) Flight tests of an open loop higher harmonic control system on an S-76A helicopter. In: 42nd Annual Forum of the American Helicopter Seciett. Washington. D.C.

49. Yu, Y. H., Gmelin, B., Heller, H., Philippe. J. J., Mercker. E. and Preisser. J. S. (1994) HHC acroacoustics rolor test at the DNW the joint German/French U.S. HART project. In: 20th European Rotorcaft Forum. Amsterdam. The Netherlands 
- 